\title{
Lomax inverse Weibull model: properties, applications, and a modified Chi-squared goodness-of-fit test for validation
}

\author{
Hafida Goual ${ }^{a, *}$, Haitham M. Yousof ${ }^{\mathrm{b}}$, M. Masoom Alic \\ a Laboratory of Probability and Statistics, University of Badji Mokhtar, Annaba, Algeria. \\ ${ }^{b}$ Department of Statistics, Mathematics and Insurance, Benha University, Egypt. \\ ${ }^{c}$ Department of Mathematical Sciences, Ball State University, Muncie, Indiana, 47306, USA.
}

\begin{abstract}
In this paper, we introduce a new extension of the Inverse Weibull distribution along with a number of its mathematical properties. Next, we construct a modified Chi-squared goodness-of-fit test based on the Nikulin-Rao-Robson statistic for censored and complete data. We describe the theory and the mechanism of the $Y_{n}^{2}$ test statistic which can be used in survival and reliability data analysis. We use the maximum likelihood estimators based on the initial non grouped data sets. Then, we conduct numerical simulations to reinforce the results. For showing the applicability of our model in various fields, we illustrate the proposed test by applications to two real data sets for complete data case and two other data sets in the presence of right censored.
\end{abstract}

Keywords: Goodness-of-Fit, NRR test, censored data, surviaval analysis, simulation, modeling, inverse Weibull, maximum likelihood.

2010 MSC: 62N03, 62E99, 62-07.

(C)2020 All rights reserved.

\section{Introduction}

The aim of this paper is to introduce first the generalization of the Inverse Weibull (IW) distribution using the Lomax-G (Lx-G) family originally introduced by Cordeiro et al. [9]. We then construct a modified Chi-squared goodness-of-fit test based on the Nikulin-Rao-Robson statistic for censored and complete data. The probability density function (PDF) and cumulative distribution function (CDF) of the IW distribution are given by (for $x \geqslant 0$ )

$$
\mathbf{h}(x ; a, b)=b a^{b} x^{-(b+1)} \exp \left[-\left(a x^{-1}\right)^{b}\right] \text { and } \mathbf{H}(x, a, b)=\exp \left[-\left(a x^{-1}\right)^{b}\right]
$$

\footnotetext{
${ }^{*}$ Corresponding author

Email addresses: goual .hafida@gmail.com (Hafida Goual), haitham . yousof@fcom . bu .edu.eg (Haitham M. Yousof), mali@bsu.edu (M. Masoom Ali)
}

doi: $10.22436 /$ jnsa.013.06.04

Received: 2019-08-29 Revised: 2019-10-29 Accepted: 2020-01-16 
respectively, where $a>0$ is a scale parameter and $b>0$ is a shape parameter. Consider the Lx-G family of distributions defined by

$$
\mathrm{F}(\mathrm{x} ; \beta, \underline{\varphi})=1-\left[1+\frac{\mathbf{H}(x ; \underline{\varphi})}{1-\mathbf{H}(x ; \underline{\varphi})}\right]^{-\beta},
$$

where $\varphi$ is the parameters vector and $\mathbf{H}(x ; \varphi)$ is the CDF of the base line distribution. The PDF corresponding to (1.2) is given by

$$
f(x ; \beta, \underline{\varphi})=\beta \frac{\mathbf{h}(x ; \varphi)}{[1-\mathbf{H}(x ; \underline{\varphi})]^{2}}\left[1+\frac{\mathbf{H}(x ; \underline{\varphi})}{1-\mathbf{H}(x ; \underline{\varphi})}\right]^{-\beta-1},
$$

where $\mathbf{h}(x ; \underline{\varphi)}) \frac{\mathrm{d}}{\mathrm{d} \chi} \mathbf{H}(\chi ; \underline{\varphi})$ is the baseline density. The hazard rate function (hrf) of $\mathrm{X}$ reduces to

$$
\tau(x)=\beta \frac{\mathbf{h}(x)}{[1-\mathbf{H}(x)]^{2}}\left[1+\frac{\mathbf{H}(x)}{1-\mathbf{H}(x)}\right]^{-1} .
$$

Inserting (1.1) in to (1.2) we have

$$
F(x ; \beta, a, b)=1-\left\{1+\frac{\exp \left[-\left(a x^{-1}\right)^{b}\right]}{1-\exp \left[-\left(a x^{-1}\right)^{b}\right]}\right\}^{-\beta}
$$

Equation (1.3) represents the CDF of the LxIW model. The PDF corresponding to (1.3) is given by

$$
f(x ; \beta, a, b)=\beta b a^{b} \frac{x^{-(b+1)} \exp \left[-\left(a x^{-1}\right)^{b}\right]}{\left\{1-\exp \left[-\left(a x^{-1}\right)^{b}\right]\right\}^{2}}\left\{1+\frac{\exp \left[-\left(a x^{-1}\right)^{b}\right]}{1-\exp \left[-\left(a x^{-1}\right)^{b}\right]}\right\}^{-\beta-1} .
$$
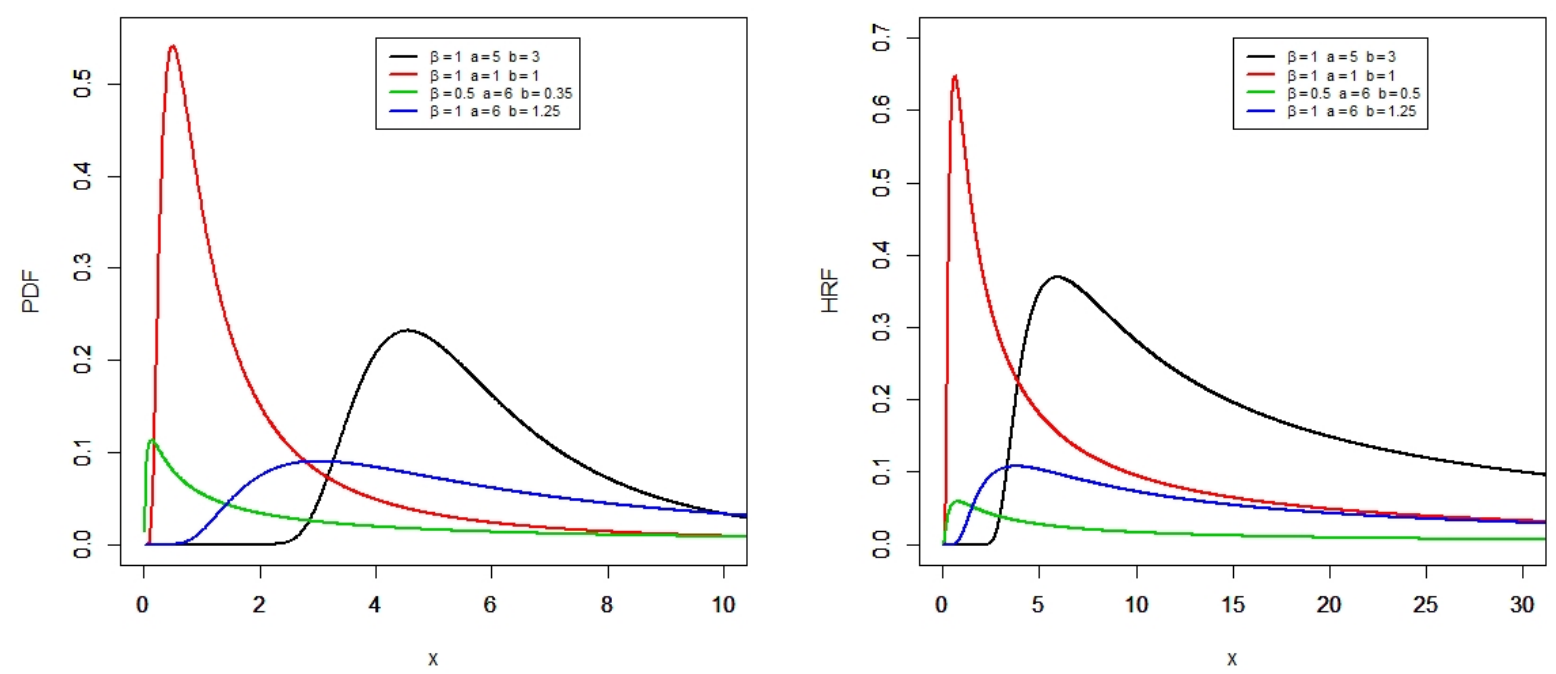

Figure 1: Plots of the LxIW PDF and HRF for selected parameter values.

The LxIW density can be right-skewed, whereas the LxIW HRF can be upside down (see Figure 1). Hereafter, we denote by $X \sim \operatorname{LxIW}(x, \beta, a, b)$ a rv having density function (1.4). The CDF (1.3) of $X$ can be 
expressed as

$$
F(x)=1-\underbrace{\left\{1+\frac{\exp \left[-\left(a x^{-1}\right)^{b}\right]}{1-\exp \left[-\left(a x^{-1}\right)^{b}\right]}\right\}^{-\beta}}_{A} .
$$

First, we consider two power series

$$
\left.\frac{1}{(1+w)^{c}}\right|_{(|w|<1, c>0)}=\sum_{h=0}^{\infty} 2^{-c-h}\left(\begin{array}{c}
-c \\
h
\end{array}\right)(w-1)^{h},
$$

and

$$
\left.\frac{1}{(1-w)^{\mathrm{c}}}\right|_{(|w|<1, \mathrm{c}>0)}=\sum_{\mathrm{h}=0}^{\infty} \frac{\Gamma(\mathrm{c}+\mathrm{h})}{\mathrm{h} ! \Gamma(\mathrm{c})} w^{\mathrm{h}} .
$$

Applying (1.6) for $A$ in (1.5) gives

$$
F(x)=1-\sum_{k_{3}=0}^{\infty} 2^{-\beta-k_{3}}\left(\begin{array}{c}
-\beta \\
k_{3}
\end{array}\right)\left\{\frac{\exp \left[-\left(a x^{-1}\right)^{b}\right]}{1-\exp \left[-\left(a x^{-1}\right)^{b}\right]}-1\right\}^{k_{3}} .
$$

Second, using the binomial expansion, the last equation can be expressed as

$$
F(x)=1-\sum_{k_{3}=0}^{\infty} \sum_{k_{1}=0}^{k_{3}} \frac{(-1)^{k_{1}}\left(\begin{array}{c}
k_{3} \\
k_{1}
\end{array}\right)\left(\begin{array}{c}
-\beta \\
k_{3}
\end{array}\right)}{2^{f i+k_{3}}\left\{\exp \left[-\left(a x^{-1}\right)^{b}\right]\right\}^{-\left(k_{3}-k_{1}\right)}} \underbrace{\left\{1-\exp \left[-\left(a x^{-1}\right)^{b}\right]\right\}^{-\left(k_{3}-k_{1}\right)}}_{\text {B }} .
$$

Third, applying (1.7) for B in the last equation we get

$$
F(x)=1-\sum_{k_{2}, k_{3}=0}^{\infty} \sum_{k_{1}=0}^{k_{3}} d_{k_{1}, k_{2}, k_{3}} \mathbf{H}_{k_{3}+k_{2}-k_{1}}(x, a, b),
$$

where

$$
d_{k_{1}, k_{2}, k_{3}}=\frac{(-1)^{k_{1}} \Gamma\left(k_{3}+k_{2}-k_{1}\right)}{2^{\beta+k_{3}} k_{2} ! \Gamma\left(k_{3}-k_{1}\right)}\left(\begin{array}{c}
k_{3} \\
k_{1}
\end{array}\right)\left(\begin{array}{c}
-\beta \\
k_{3}
\end{array}\right) .
$$

and $\mathbf{H}_{k_{3}+k_{2}-k_{1}}(x, a, b)$ is the CDF of the IW CDF with scale parameter $a\left[k_{3}+k_{2}-k_{1}\right]^{\frac{1}{b}}$ and shape parameter $b$. By differentiating (1.8), we obtain

$$
f(x)=\left.\sum_{k_{2}, k_{3}=0}^{\infty} \sum_{k_{1}=0}^{k_{3}} A_{k_{1}, k_{2}, k_{3}} \mathbf{h}_{k_{3}+k_{2}-k_{1}}(x, a, b)\right|_{\left(k_{2}+k_{3} \geqslant 1\right)},
$$

where $\mathbf{h}_{k_{3}+k_{2}-k_{1}}(x, a, b)$ is the IW density with scale parameter $a\left(k_{3}+k_{2}-k_{1}\right)^{\frac{1}{b}}$ and shape parameter $b$ and $A_{k_{1}, k_{2}, k_{3}}=-d_{k_{1}, k_{2}, k_{3}}$.

\section{Properties}

\subsection{Moments and generating function}

Let $Z$ be a random variable (rv) having the IW distribution (1.1) with parameters $a$ and $b$. For $r<b$, the $\mathrm{r}^{\text {th }}$ ordinary and incomplete moments of $Z$ are given by

$$
\mu_{r}^{\prime}=a^{r} \Gamma\left(1-\frac{r}{b}\right),
$$


and

$$
\varphi_{r}(t)=a^{r} \gamma\left(1-\frac{r}{b},\left(\frac{a}{t}\right)^{b}\right)
$$

respectively, where

$$
\begin{aligned}
\left.\Gamma(1+\boldsymbol{\sigma})\right|_{(\boldsymbol{\sigma}>0)} & =\int_{0}^{\infty} \mathrm{t}^{\boldsymbol{\sigma}} \exp (-\mathrm{t}) \mathrm{dt}, \\
\left.\Gamma(1+\boldsymbol{\sigma}, w)\right|_{(\boldsymbol{\sigma}>0)} & =\int_{w}^{\infty} \mathrm{t}^{\boldsymbol{\sigma}} \exp (-\mathrm{t}) \mathrm{dt}, \\
\left.\gamma(\boldsymbol{\sigma}, w)\right|_{(\boldsymbol{\sigma} \neq 0,-1,-2, \ldots)} & =\int_{0}^{w} \mathrm{t}^{\boldsymbol{\sigma}-1} \exp (-\mathrm{t}) \mathrm{dt}=\frac{w^{\boldsymbol{\sigma}}}{\boldsymbol{\sigma}}\left\{1 \mathbf{F}_{1}[\boldsymbol{\sigma} ; \boldsymbol{\sigma} \mathbf{a}+1 ;-\mathfrak{w}]\right\}=\sum_{\mathrm{k}_{3}=0}^{\infty} \frac{(-1)^{\mathrm{k}_{3}}}{\mathrm{k}_{3} !\left(\boldsymbol{\sigma}+\mathrm{k}_{3}\right)} w^{\boldsymbol{\sigma}+\mathrm{k}_{3},} \\
\Gamma(\boldsymbol{\sigma}, w)+\gamma(\boldsymbol{\sigma}, w) & =\Gamma(\boldsymbol{\sigma}),
\end{aligned}
$$

and ${ }_{1} \mathbf{F}_{1}[\cdot, \cdot, \cdot]$ is a confluent hypergeometric function.

The $\mathrm{r}^{\text {th }}$ ordinary moment of $X$ say $\mu_{r}^{\prime}=E\left(X^{r}\right)$, is determined from (1.9) as

$$
\mu_{r}^{\prime}=\left.\sum_{k_{2}, k_{3}=0}^{\infty} \sum_{k_{1}=0}^{k_{3}} A_{k_{1}, k_{2}, k_{3}} \frac{\left[k_{3}+k_{2}-k_{1}\right]^{r / b}}{a^{-r}} \Gamma\left(1-\frac{r}{b}\right)\right|_{\left(k_{2}+k_{3} \geqslant 1, r<b\right)} .
$$

The $\mathrm{r}^{\text {th }}$ incomplete moment of $X$, say $\varphi_{r}(t)$, can be determined from (1.9) as

$$
\varphi_{r}(t)=\int_{-\infty}^{t} x^{r} f(x) d x=\left.\sum_{\substack{k_{2}, k_{3}=0 \\ \mid\left(k_{2}+k_{3} \geqslant 1\right)}}^{\infty} \sum_{k_{1}=0}^{k_{3}} A_{k_{1}, k_{2}, k_{3}} \frac{\left[k_{3}+k_{2}-k_{1}\right]^{r / b}}{a^{-r}} \gamma\left(1-\frac{r}{b},\left(\frac{a}{t}\right)^{b}\right)\right|_{(r<b)} .
$$

The moment generating function (mgf) $M(t)=E\left(e^{t X}\right)$ of $X$ follows from (1.9) as

$$
M(t)=\left.\sum_{k_{2}, k_{3}, r}^{\infty} \sum_{k_{1}=0}^{k_{3}} A_{k_{1}, k_{2}, k_{3}} \frac{t^{r}\left[k_{3}+k_{2}-k_{1}\right]^{r / b}}{r ! a^{-r}} \Gamma\left(1-\frac{r}{b}\right)\right|_{\left(k_{2}+k_{3} \geqslant 1, r<b\right.} .
$$

\subsection{Probability weighted moments}

The PWMs are generally used for estimating parameters of a distribution whose inverse form cannot be expressed explicitly. The $(s, r)^{\text {th }}$ PWM of $X$ denoted by $\rho_{s, r}$ is formally defined by

$$
\rho_{s, r}=\mathbf{E}\left\{X^{s} F(X)^{r}\right\}=\int_{-\infty}^{\infty} x^{s} F(x)^{r} f(x) d x
$$

Using (1.3), we have

$$
F(x)^{r}=\left[1-\left(1+\left\{\frac{\exp \left[-\left(a x^{-1}\right)^{b}\right]}{1-\exp \left[-\left(a x^{-1}\right)^{b}\right]}\right\}\right)^{-\beta}\right]^{r}
$$

Consider the Taylor series

$$
z^{\beta}=\sum_{h=0}^{\infty} \frac{(\beta)_{h}}{h !}(z-1)^{h}=\sum_{k_{1}=0}^{\infty} f_{k_{1}}(\beta) z^{k_{1}}
$$

where

$$
(\beta)_{h}=\beta(\beta-1) \cdots(\beta-h+1)
$$


is the descending factorial and

$$
f_{k_{1}}(\beta)=\sum_{h=k_{1}}^{\infty} \frac{(-1)^{h-k_{1}}(\beta)_{h}}{h !}\left(\begin{array}{l}
h \\
k_{1}
\end{array}\right) .
$$

First, applying the Taylor series in $z^{\beta}$ for $F(x)^{r}$, we obtain

$$
F(x)^{r}=\sum_{k_{1}=0}^{\infty}(-1)^{k_{1}} f_{k_{1}}(r)\left\{1+\frac{\exp \left[-\left(a x^{-1}\right)^{b}\right]}{1-\exp \left[-\left(a x^{-1}\right)^{b}\right]}\right\}^{-k_{1} \beta} .
$$

Second, using (1.4) and the last equation, we have

$$
f(x) F(x)^{r}=\beta b a^{b} \frac{x^{-(b+1)} \exp \left[-\left(a x^{-1}\right)^{b}\right]}{\left\{1-\exp \left[-\left(a x^{-1}\right)^{b}\right]\right\}^{2}} \sum_{k_{1}=0}^{\infty}(-1)^{k_{1}} f_{k_{1}}(r) \underbrace{\left\{1+\frac{\exp \left[-\left(a x^{-1}\right)^{b}\right]}{1-\exp \left[-\left(a x^{-1}\right)^{b}\right]}\right\}^{-\left(k_{1}+1\right) \beta-1}}_{c} .
$$

Applying (1.6) for $\mathrm{C}$ in the last equation, we obtain

$$
\begin{aligned}
f(x) F(x)^{r}= & \sum_{k_{1}, k_{3}=0}^{\infty} \frac{(-1)^{k_{1}} f_{k_{1}}(r) \beta b a^{b} \exp \left[-\left(a x^{-1}\right)^{b}\right]}{2^{\left(k_{1}+1\right) \beta+k_{3}+1} x^{b+1}\left\{1-\exp \left[-\left(a x^{-1}\right)^{b}\right]\right\}^{2}} \\
& \times \underbrace{\left\{\frac{\exp \left[-\left(a x^{-1}\right)^{b}\right]}{1-\exp \left[-\left(a x^{-1}\right)^{b}\right]}-1\right\}^{k_{3}}\left(\begin{array}{c}
-\left(k_{1}+1\right) \beta-1 \\
k_{3}
\end{array}\right) .}_{D} .
\end{aligned}
$$

Third, using the binomial expansion for $\mathrm{D}$, the last equation can be rewritten as

$$
\begin{aligned}
f(x) F(x)^{r}= & \beta g(x) \sum_{k_{1}, k_{3}=0}^{\infty} \sum_{k_{2}=0}^{k_{3}}(-1)^{k_{1}+k_{2}} 2^{-\left(k_{1}+1\right) \beta-k_{3}-1}\left(\begin{array}{l}
k_{3} \\
k_{2}
\end{array}\right)\left(\begin{array}{c}
-\left(k_{1}+1\right) \beta-1 \\
k_{3}
\end{array}\right) \\
& \times f_{k_{1}}(r)\left\{\exp \left[-\left(a x^{-1}\right)^{b}\right]\right\}^{k_{3}-k_{2}} \underbrace{\left\{1-\exp \left[-\left(a x^{-1}\right)^{b}\right]\right\}^{-\left[k_{3}-k_{2}+2\right]}}_{E} .
\end{aligned}
$$

Applying (1.7) for $E$ in the last equation gives

$$
f(x) F(x)^{r}=\sum_{k_{3}, m=0}^{\infty} \sum_{k_{2}=0}^{k_{3}} w_{k_{2}, k_{3}, m}^{(r)} \mathbf{B}_{k_{3}-k_{2}+m+1}(x ; a, b),
$$

where $w_{k_{2}, k_{3}, m}^{(r)}=\beta v_{k_{2}, k_{3}, m} f_{k_{1}}(r), f_{k_{1}}(r)$ as defined in (2.1) and

$$
v_{k_{2}, k_{3}, m}=\left.\sum_{k_{1}=0}^{\infty} \frac{(-1)^{k_{1}+k_{2}}\left(k_{3}-k_{2}+2\right)^{(m)}\left(\begin{array}{l}
k_{3} \\
k_{2}
\end{array}\right)\left(\begin{array}{c}
-\left(k_{1}+1\right) f i-1 \\
k_{3}
\end{array}\right)}{2^{\left(k_{1}+1\right) \beta+k_{3}+1}\left[k_{3}-k_{2}+m+1 m !\right.}\right|_{\left(k_{2} \leqslant k_{3}\right)},
$$

where $a^{(n)}=\Gamma(a+n) / \Gamma(a)$ denotes the rising factorial. Finally, the $(s, r)^{\text {th }}$ PWM of $X$ can be determined as

$$
\rho_{s, r}=\left.\sum_{k_{3}, m=0}^{\infty} \sum_{k_{2}=0}^{k_{3}} w_{k_{2}, k_{3}, m}^{(r)} \frac{\left[k_{3}-k_{2}+m+1\right]^{s / b}}{a^{-s}} \Gamma\left(1-\frac{s}{b}\right)\right|_{\left(k_{2} \leqslant k_{3}, s<b\right)} .
$$


2.3. Residual life and reversed residual life functions

The $\mathrm{n}^{\text {th }}$ moment of the residual life, say

$$
m_{n}(t)=\left.E\left[(X-t)^{n}\right]\right|_{(X>t, n=1,2, \ldots)},
$$

uniquely determines $F(x)$. The $n^{\text {th }}$ moment of the residual life of $X$ is given by

$$
m_{n}(t)=\frac{\int_{t}^{\infty}(x-t)^{n} d F(x)}{1-F(t)}
$$

Therefore

$$
m_{n}(t)=\left.\frac{1}{1-F(t)} \sum_{k_{2}, k_{3}=0}^{\infty} \sum_{k_{1}=0}^{k_{3}} A_{k_{1}, k_{2}, k_{3}}^{(m)} \frac{\left[k_{3}+k_{2}-k_{1}\right]^{n / b}}{a^{-n}} \Gamma\left(1-\frac{n}{b},\left(\frac{a}{t}\right)^{b}\right)\right|_{\left(k_{2}+k_{3} \geqslant 1, n<b\right)}
$$

where

$$
A_{k_{1}, k_{2}, k_{3}}^{(m)}=A_{k_{1}, k_{2}, k_{3}}(1-t)^{n} .
$$

The mean residual life (MRL) function or the life expectation at age $t$ is defined by

$$
\left.\mathrm{m}_{1}(\mathrm{t})\right|_{(X>t, n=1)}=\mathbf{E}[(X-t)]
$$

which represents the expected additional life length for a unit which is alive at age $t$. The MRL of $X$ can be obtained by setting $n=1$ in the last equation. The $n^{\text {th }}$ moment of the reversed residual life, say

$$
M_{n}(t)=\left.E\left[(t-X)^{n}\right]\right|_{(t>0, X \leqslant t, n=1,2, \ldots)},
$$

uniquely determines $F(x)$. We obtain

$$
M_{n}(t)=\frac{\int_{0}^{t}(t-x)^{n} d F(x)}{F(t)}
$$

Then, the $\mathrm{n}^{\text {th }}$ moment of the reversed residual life of $X$ is

$$
M_{n}(t)=\left.\frac{1}{F(t)} \sum_{k_{2}, k_{3}=0}^{\infty} \sum_{k_{1}=0}^{k_{3}} A_{k_{1}, k_{2}, k_{3}}^{(M)} \frac{\left[k_{3}+k_{2}-k_{1}\right]^{n / b}}{a^{-n}} a\left(1-\frac{n}{b},\left(\frac{a}{t}\right)^{b}\right)\right|_{\left(k_{2}+k_{3} \geqslant 1, n<b\right),}
$$

where

$$
A_{k_{1}, k_{2}, k_{3}}^{(M)}=A_{k_{1}, k_{2}, k_{3}} \sum_{r=0}^{n}(-1)^{r}\left(\begin{array}{l}
n \\
r
\end{array}\right) t^{n-r} .
$$

The mean inactivity time (MIT), also called the mean reversed residual life function, is given by

$$
M_{1}(t)=\left.E[(t-X)]\right|_{(t>0, X \leqslant t, n=1)},
$$

and it represents the waiting time elapsed since the failure of an item on condition that this failure had occurred in $(0, t)$. The MIT of the new model is obtained easily by setting $n=1$ in the above equation.

\subsection{Order statistics}

Order statistics make their appearance in many areas of statistical theory and practice. Let $X_{1}, \ldots, X_{n}$ be a random sample (RS) from the LxIW and let $X_{1: n}, \ldots, X_{n: n}$ be the corresponding order statistics. The 
PDF of the $i^{\text {th }}$ order statistic, say $X_{i: n}$, is given by

$$
f_{i: n}(x)=\frac{f(x)}{B(i, n-i+1)} \sum_{r=0}^{n-i}(-1)^{r}\left(\begin{array}{c}
n-i \\
r
\end{array}\right) F^{r+i-1}(x),
$$

where $\mathrm{B}(\cdot, \cdot)$ is the beta function, then we can write

$$
f(x) F(x)^{r+i-1}=\sum_{k_{3}, m=0}^{\infty} \sum_{j=0}^{k_{3}} w_{j, k_{3}, m}^{(r+i-1)} \boldsymbol{B}_{\left(k_{3}-j+m+1\right.}(x ; a, b),
$$

where $w_{j, k_{3}, m}^{(r+i-1)}$ is as defined before. So, the PDF of $X_{i: n}$ becomes using the last expression as

$$
f_{i: n}(x)=\frac{1}{B(i, n-i+1)} \sum_{k_{3}, m=0}^{\infty} \sum_{r=0}^{n-i} \sum_{j=0}^{k_{3}}(-1)^{r}\left(\begin{array}{c}
n-i \\
r
\end{array}\right) w_{j, k_{3}, m}^{(r+i-1)} B_{k_{3}-j+m+1}(x ; a, b) .
$$

Then, the density function of the LxIW order statistics is a linear combination of the IW density. Based on this equation, the properties of $X_{i: n}$ can be easily determined from those properties of the IW density. Then The $\mathrm{q}^{\text {th }}$ ordinary moment of $X_{i: n}$ say $E\left(X_{i: n}^{w}\right)$, is determined from (2.2) as

$$
\mathbf{E}\left(X_{i: n}^{w}\right)=\left.\sum_{k_{3}, m}^{\infty} \sum_{r=0}^{n-i} \sum_{j=0}^{k_{3}} \frac{(-1)^{r}\left(\begin{array}{c}
n-i \\
r
\end{array}\right) w_{j, k_{3}, m}^{(r+i-1)}}{B(i, n-i+1)} a^{w}\left[k_{3}-j+m+1\right]^{\frac{w}{b}} \Gamma\left(1-\frac{w}{b}\right)\right|_{(w<b)} .
$$

\section{Maximum likelihood estimation in case of complete data}

\subsection{Maximum likelihood estimation}

Let $x_{1}, \ldots, x_{n}$ be a RS from the LxIW model with parameters $\beta, a$ and $b$. Let $\Theta=(\beta, a, b)^{\top}$ be the $3 \times 1$ parameter vector. For determining the MLE of $\Theta$, we have the log-likelihood function

$$
\ell=\ell(\Theta)=n \log \beta+n \log b+n b \log a-(b+1) \sum_{i=1}^{n} x_{i}-\sum_{i=1}^{n}\left(a x^{-1}\right)^{b}-2 \sum_{i=1}^{n} \log \left(1-s_{i}\right)-(\beta+1) \sum_{i=1}^{n} \log z_{i},
$$

where

$$
s_{i}=\exp \left[-\left(a x^{-1}\right)^{b}\right] \text { and } z_{i}=\left[1+\left(\frac{s_{i}}{1-s_{i}}\right)\right] .
$$

The score vector $\mathbf{I}_{(\Theta)}=\frac{\partial \ell}{\partial \Theta}=\left(\frac{\partial \ell}{\partial \mathrm{fi}}, \frac{\partial \ell}{\partial \mathrm{a}}, \frac{\partial \ell}{\partial \mathrm{b}}\right)^{\top}$ is given as

$$
\begin{aligned}
& \mathbf{I}_{(\beta)}=\frac{n}{\beta}-\sum_{i=1}^{n} \log z_{i}, \\
& \mathbf{I}_{(a)}=\frac{n b}{a}-b \sum_{i=1}^{n} \frac{1}{x_{i}}\left(a x^{-1}\right)^{b-1}-2 b \sum_{i=1}^{n} \frac{\left(a x^{-1}\right)^{b-1} \frac{1}{x_{i}} s_{i}}{1-s_{i}}-(\beta+1) \sum_{i=1}^{n} \frac{m_{i}}{z_{i}}, \\
& \mathbf{I}_{(b)}=\frac{n}{b}+n \log a-\sum_{i=1}^{n} x_{i}-\sum_{i=1}^{n} w_{i}-2 \sum_{i=1}^{n} \frac{w_{i} s_{i}}{1-s_{i}}-(\beta+1) \sum_{i=1}^{n} \frac{t_{i}}{z_{i}},
\end{aligned}
$$

where

$$
m_{i}=-b \frac{s_{i}}{x_{i}}\left(a x^{-1}\right)^{b-1}\left[1-s_{i}\right]^{-2}, \quad t_{i}=-\frac{w_{i} s_{i}}{\left[1-s_{i}\right]^{2}}, \quad \text { and } \quad w_{i}=\left(a x^{-1}\right)^{b} \log \left(a x^{-1}\right) .
$$

Setting the nonlinear system of equations $\mathbf{I}_{(\beta)}=0, \mathbf{I}_{(\mathrm{a})}=0$ and $\mathbf{I}_{(\mathrm{b})}=0$ and solving them simultaneously yields the MLE $\widehat{\Theta}=(\widehat{\beta}, \widehat{a}, \widehat{b})^{\top}$. To solve these equations, it is usually more convenient to use nonlinear optimization methods such as the quasi-Newton algorithm to numerically maximize $\ell$. 


\subsection{Data analysis: case of complete data}

This section presents two applications of the LxIW distribution using real data sets. We shall compare the fit of the new distribution with the WIW (Afify et al. [1])), exponentiated IW (EIW) (Nadarajah and Kotz [26]), Kumaraswamy IW (KumIW) (Mead [23]), beta IW (BIW) (Nadarajah and Gupta [25]), transmuted IW (TIW) (Mahmoud and Mandouh, [22]), gamma extended IW (GEIW) (da Silva et. al. [11]), Marshall-Olkin IW (MOIW) (Krishna et al. [21]) and IW distributions with corresponding densities (for $x>0$ ). The unknown parameters of the above pdfs are all positive real numbers except for the TFr distribution for which $|a| \leqslant 1$. The first data set consists of 100 observations of breaking stress of carbon fbres given by Nichols and Padgett [27]. The data are: 0.92, 0.928, 0.997, 0.9971, 1.061, 1.117, 1.162, 1.183, $1.187,1.192,1.196,1.213,1.215,1.2199,1.22,1.224,1.225,1.228,1.237,1.24,1.244,1.259,1.261,1.263,1.276$, $1.31,1.321,1.329,1.331,1.337,1.351,1.359,1.388,1.408,1.449,1.4497,1.45,1.459,1.471,1.475,1.477,1.48$, $1.489,1.501,1.507,1.515,1.53,1.5304,1.533,1.544,1.5443,1.552,1.556,1.562,1.566,1.585,1.586,1.599$, $1.602,1.614,1.616,1.617,1.628,1.684,1.711,1.718,1.733,1.738,1.743,1.759,1.777,1.794,1.799,1.806,1.814$, $1.816,1.828,1.83,1.884,1.892,1.944,1.972,1.984,1.987,2.02,2.0304,2.029,2.035,2.037,2.043,2.046,2.059$, $2.111,2.165,2.686,2.778,2.972,3.504,3.863,5.306$. Figure 2 gives the TTT plots for the first data set. From Figure 2 we note that the empirical HRFs of the data is increasing.

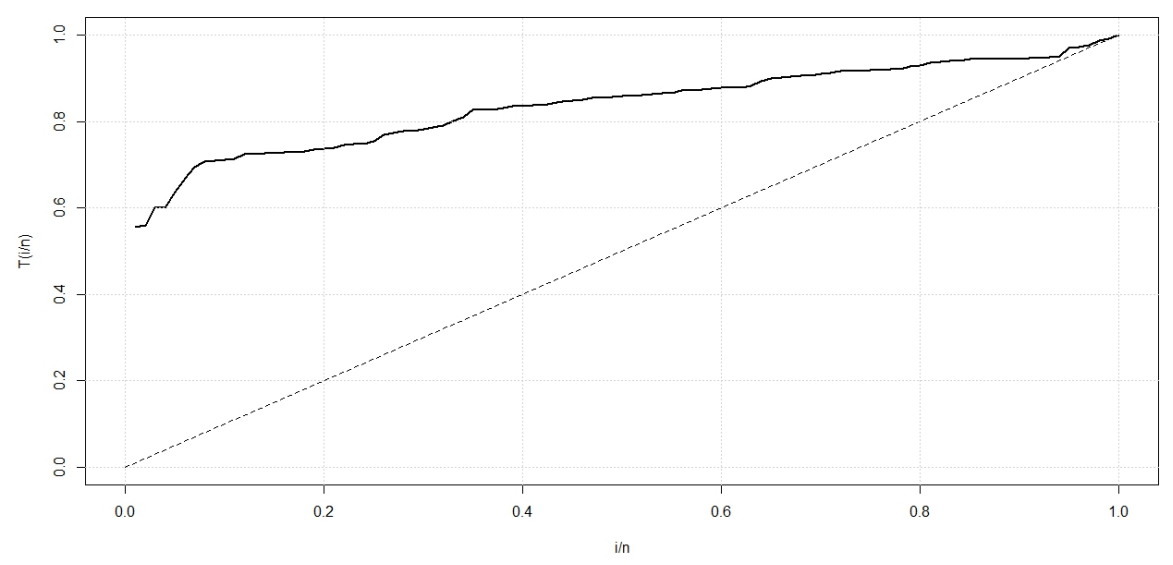

Figure 2: TTT plots for the first data set.

The second data set (Smith and Naylor [31]) consists of 63 observations of the strengths of $1.5 \mathrm{~cm}$ glass fibres, originally obtained by workers at the UK National Physical Laboratory. Unfortunately, the units of measurement are not given in the paper. The data are: 1.014, 1.081, 1.082, 1.185, 1.223, 1.248, 1.267, 1.271, $1.272,1.275,1.276,1.278,1.286,1.288,1.292,1.304,1.306,1.355,1.361,1.364,1.379,1.409,1.426,1.459,1.46$, $1.476,1.481,1.484,1.501,1.506,1.524,1.526,1.535,1.541,1.568,1.579,1.581,1.591,1.593,1.602,1.666,1.67$, $1.684,1.691,1.704,1.731,1.735,1.747,1.748,1.757,1.800,1.806,1.867,1.876,1.878,1.91,1.916,1.972,2.012$, $2.456,2.592,3.197,4.121$. Figure 3 gives the TTT plots for the first data set. From Figure 3 we note that the empirical HRFs of the data is also increasing.

In order to compare the distributions, we consider the following criteria: the $-2 \widehat{\ell}$ (Maximized LogLikelihood), AIC (Akaike Information Criterion), CAIC (Consistent Akaike Information Criterion), BIC (Bayesian Information Criterion) and HQIC (Hannan-Quinn Information Criterion). These statistics are given by

$$
\begin{aligned}
\text { AIC } & =-2 \widehat{\ell}+2 k_{(p)}, \\
\text { BIC } & =-2 \widehat{\ell}+k_{(p)} \log (n), \\
\text { HQIC } & =-2 \widehat{\ell}+2 k_{(p)} \log [\log (n)],
\end{aligned}
$$


and

$$
\mathrm{CAIC}=-2 \widehat{\ell}+2 k_{(p)} n /(n-k-1)
$$

where $\widehat{\ell}$ denotes the log-likelihood function evaluated at the MLEs, $k_{(p)}$ is the number of model parameters and $n$ is the sample size. The model with minimum values for these statistics could be chosen as the best model to fit the data. All results are obtained using the R PROGRAM.

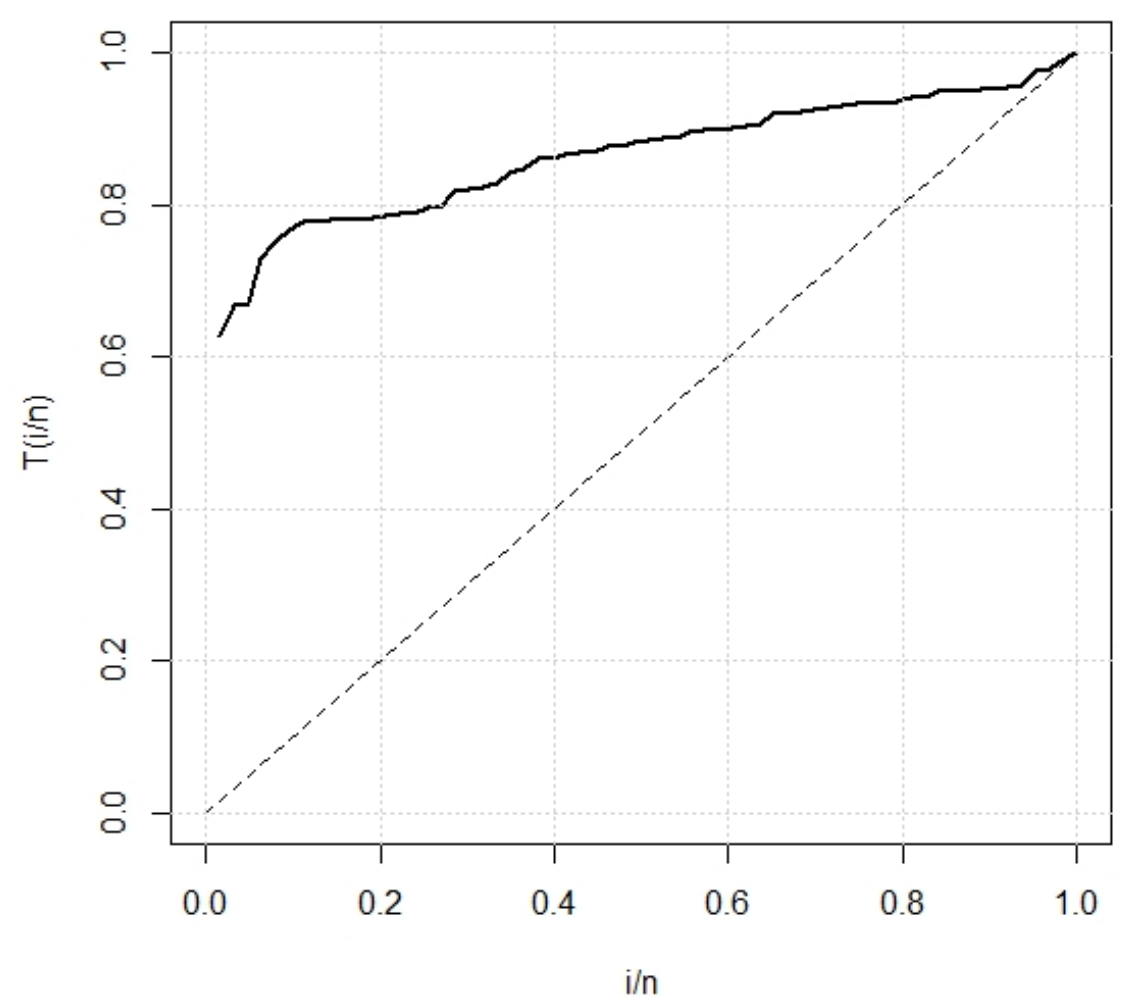

Figure 3: TTT plots for the second data set.

Table 1: The statistics $-2 \widehat{\ell}$, AIC, BIC, HQIC and CAIC for breaking stress of carbon fibre data.

\begin{tabular}{cccccc}
\hline Model & \multicolumn{5}{c}{ Goodness of fit criteria } \\
\cline { 2 - 6 } & $-2 \ell$ & AIC & BIC & HQIC & CAIC \\
\hline LxIW & $\mathbf{1 0 5 . 8}$ & $\mathbf{1 1 1 . 9}$ & $\mathbf{1 1 9 . 7 6}$ & $\mathbf{1 1 5 . 1}$ & $\mathbf{1 1 1 . 9}$ \\
WIW & 286.5 & 294.5 & 304.9 & 298.7 & 294.9 \\
EIW & 289.7 & 295.7 & 303.5 & 298.9 & 296.0 \\
KumIW & 289.1 & 297.1 & 307.5 & 301.3 & 297.5 \\
BIW & 303.1 & 311.1 & 321.6 & 315.4 & 311.6 \\
GEIW & 304 & 312 & 332.4 & 316.2 & 312.4 \\
IW & 344.3 & 348.3 & 353.5 & 350.4 & 348.4 \\
TIW & 344.5 & 350.5 & 358.3 & 353.6 & 350.7 \\
MOIW & 345.3 & 351.3 & 359.1 & 354.5 & 351.6 \\
\hline
\end{tabular}


Table 2: MLEs and their standard errors for breaking stress data.

\begin{tabular}{|c|c|c|c|c|}
\hline \multirow[t]{2}{*}{ Model } & \multicolumn{4}{|c|}{ Estimates } \\
\hline & $\widehat{\alpha}$ & $\widehat{\beta}$ & $\widehat{a}$ & $\widehat{b}$ \\
\hline LxIW & & $\begin{array}{c}1.629 \\
(0.697)\end{array}$ & $\begin{array}{c}1.558 \\
(0.1695)\end{array}$ & $\begin{array}{c}3.421 \\
(0.763)\end{array}$ \\
\hline WIW & $\begin{array}{c}2.2231 \\
(11.409)\end{array}$ & $\begin{array}{c}0.355 \\
(0.411)\end{array}$ & $\begin{array}{c}6.9721 \\
(113.811)\end{array}$ & $\begin{array}{l}4.9179 \\
(3.756)\end{array}$ \\
\hline $\mathrm{EF}$ & $\begin{array}{l}69.1489 \\
(57.349)\end{array}$ & $\begin{array}{c}0.5019 \\
(0.08)\end{array}$ & $\begin{array}{l}145.3275 \\
(122.924)\end{array}$ & \\
\hline KumIW & $\begin{array}{l}2.0556 \\
(0.071)\end{array}$ & $\begin{array}{c}0.4654 \\
(0.00701)\end{array}$ & $\begin{array}{c}6.2815 \\
(0.063)\end{array}$ & $\begin{array}{l}224.18 \\
(0.164)\end{array}$ \\
\hline BIW & $\begin{array}{l}1.6097 \\
(2.498)\end{array}$ & $\begin{array}{l}0.4046 \\
(0.108)\end{array}$ & $\begin{array}{l}22.0143 \\
(21.432)\end{array}$ & $\begin{array}{l}29.7617 \\
(17.479)\end{array}$ \\
\hline GEIW & $\begin{array}{l}1.3692 \\
(2.017)\end{array}$ & $\begin{array}{l}0.4776 \\
(0.133)\end{array}$ & $\begin{array}{l}27.6452 \\
(14.136)\end{array}$ & $\begin{array}{c}17.4581 \\
(14.818)\end{array}$ \\
\hline IW & $\begin{array}{c}1.8705 \\
(0.112)\end{array}$ & $\begin{array}{l}1.7766 \\
(0.113)\end{array}$ & & \\
\hline TIW & $\begin{array}{c}1.9315 \\
(0.097)\end{array}$ & $\begin{array}{c}1.7435 \\
(0.076)\end{array}$ & $\begin{array}{l}0.0819 \\
(0.198)\end{array}$ & \\
\hline MOIW & $\begin{array}{l}2.3066 \\
(0.498)\end{array}$ & $\begin{array}{c}1.5796 \\
(0.16)\end{array}$ & $\begin{array}{c}0.5988 \\
(0.3091)\end{array}$ & \\
\hline
\end{tabular}
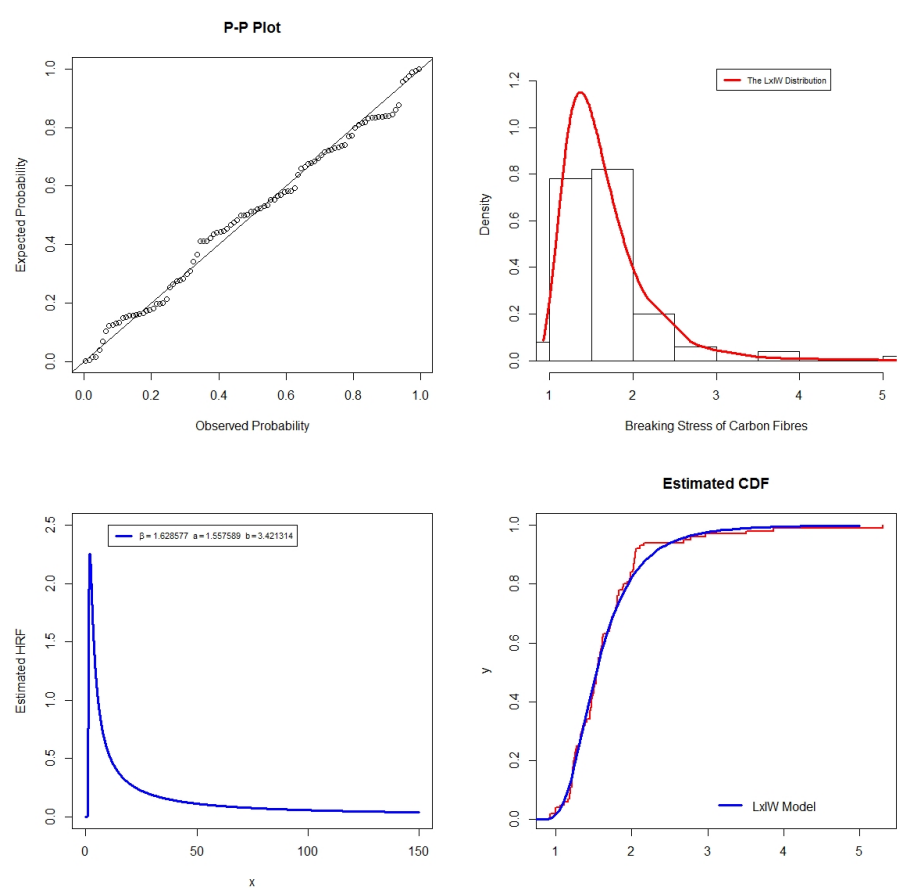

Figure 4: P-P plot, estimated PDF, estimated HRF and estimated CDF for first data set. 
Table 3: The statistics $-2 \widehat{\ell}, A I C, B I C, H Q I C$ and CAIC values for strengths of $1.5 \mathrm{~cm}$ glass fibre data.

\begin{tabular}{|cccccc|}
\hline Model & \multicolumn{5}{c|}{ Measures } \\
& $-2 \widehat{\ell}$ & AIC & BIC & HQIC & CAIC \\
\hline LxIW & 39.8 & $\mathbf{4 5 . 8 7}$ & $\mathbf{5 2 . 3}$ & $\mathbf{4 8 . 4}$ & $\mathbf{4 6 . 2 7}$ \\
KumIW & 39.9 & 47.6 & 56.2 & 51 & 48.3 \\
EIW & 44.3 & 50.5 & 56.7 & 52.8 & 50.7 \\
BIW & 60.6 & 68.6 & 77.2 & 72.0 & 69.3 \\
GEIW & 61.6 & 69.6 & 78.1 & 72.9 & 70.3 \\
IW & 93.7 & 97.7 & 102 & 99.4 & 97.9 \\
TIW & 94.1 & 100.1 & 106.5 & 102.6 & 100.5 \\
MOIW & 95.7 & 101.7 & 108.2 & 104.2 & 102.1 \\
\hline
\end{tabular}

Table 4: MLEs and their standard errors for strengths data.

\begin{tabular}{|ccccc|}
\hline Model & \multicolumn{4}{c|}{ Estimates } \\
\cline { 2 - 5 } & $\widehat{\alpha}$ & $\widehat{\beta}$ & $\widehat{\mathrm{a}}$ & $\widehat{\mathrm{b}}$ \\
\hline LxIW & & 1.283 & 1.472 & 4.77 \\
& & $(0.639)$ & $(0.137)$ & $(1.312)$ \\
KumIW & 2.116 & 0.740 & 5.504 & 857.343 \\
& $(4.555)$ & $(0.071)$ & $(7.982)$ & $(153.948)$ \\
EIW & 7.816 & 0.999 & 132.827 & \\
& $(2.945)$ & $(0.136)$ & $(116.63)$ & \\
& & & & \\
BIW & 2.0518 & 0.6466 & 15.0756 & 36.9397 \\
& $(0.986)$ & $(0.163)$ & $(12.057)$ & $(22.649)$ \\
& & & & \\
GEIW & 1.6625 & 0.7421 & 32.112 & 13.2688 \\
& $(0.952)$ & $(0.197)$ & $(17.397)$ & $(9.967)$ \\
& & & & \\
IW & 1.264 & 2.888 & & \\
& $(0.059)$ & $(0.234)$ & & \\
TIW & 1.3068 & 2.7898 & 0.1298 & \\
& $(0.034)$ & $(0.165)$ & $(0.208)$ & \\
MOIW & 1.5441 & 2.3876 & 0.4816 & \\
& $(0.226)$ & $(0.253)$ & $(0.252)$ & \\
\hline
\end{tabular}

Tables 2 and 4 give the MLEs and their standard errors for two data sets. Tables 1 and 3 compare the LxIW model with the WIW, KIW, EIW, BIW, GEIW, TMOIW, TIW, MOIW and IW distributions. The LxIW model gives the lowest values for the AIC, BIC, HQIC and CAIC statistics (in bold values) among all fitted models to these data. So, it could be chosen as the best model among them. Figure 4 displays P-P plot, estimated density, estimated HRF and estimated CDF of the new model for the first data. Figure 5 displays P-P plot, estimated density, estimated HRF and estimated CDF of the new model for the second data. These plots reveal that the proposed distribution yields a better fit than other nested and non-nested models for both data sets. 

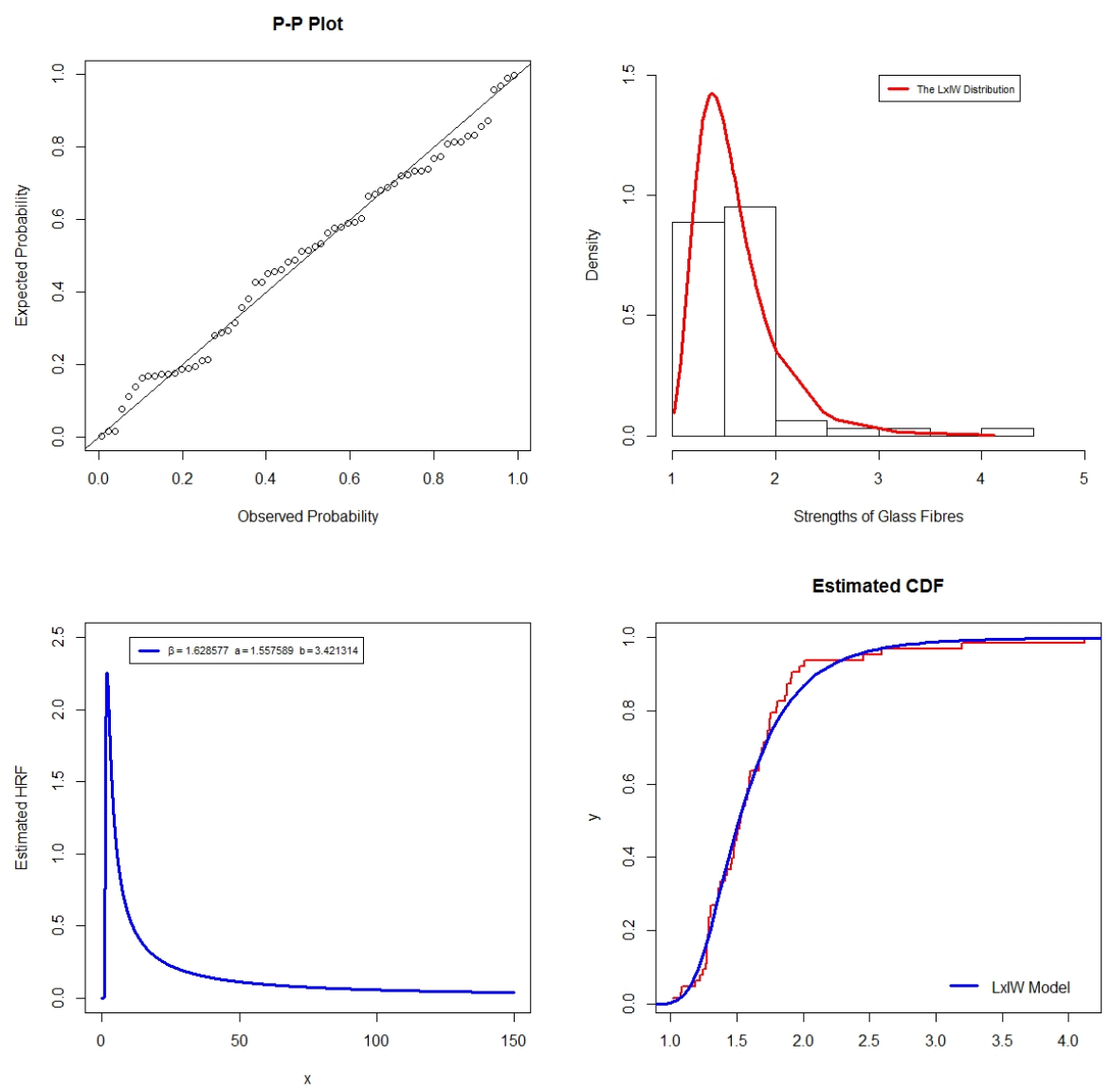

Figure 5: P-P plot, estimated PDF, estimated HRF and estimated CDF for second data set.

\subsection{Simulations: case of complete data}

We consider the LxIW model. The data were simulated $N=10,000$ times; with parameter values $\beta=1.2, a=2.5, b=0.8$ and sample sizes $n=30, n=100, n=150$ and $n=300$. Using the $R$ software and the Barzilai-Borwein (BB) algorithm (Ravi [32]) for calculating the averages of the simulated values of the maximum likelihood estimators $\widehat{\beta}, \widehat{a}, \widehat{b}$ parameters and their mean squared errors (MSEs). Results are presented in Table 5. From Table 5, we observe that the maximum likelihood estimates for the LxIW model are convergent.

Table 5: Maximum likelihood estimates $(\widehat{\beta}, \widehat{a}, \widehat{b})$ of the parameters and their MSEs.

\begin{tabular}{|c|c|c|c|c|}
\hline $\mathrm{N}=10000$ & $\mathrm{n}=30$ & $\mathrm{n}=100$ & $\mathrm{n}=150$ & $\mathrm{n}=300$ \\
\hline$\widehat{\beta}$ & 1.30024 & 1.25143 & 1.24557 & 1.21403 \\
\hline $\mathrm{MSE}$ & $5.38 .10^{-4}$ & $4.51 .10^{-4}$ & $1.98 .10^{-05}$ & $3.01 .10^{-05}$ \\
\hline$\widehat{\mathrm{a}}$ & 2.4851 & 2.4805 & 2.4911 & 2.51098 \\
\hline $\mathrm{MSE}$ & $2.52 .10^{-4}$ & 0.00267 & 0.00141 & 0.00032 \\
\hline$\widehat{\mathrm{b}}$ & 0.91682 & 0.90048 & 0.82410 & 0.81016 \\
\hline $\mathrm{MSE}$ & 0.049871 & 0.02447 & 0.0021 & $2.95 .10^{-4}$ \\
\hline
\end{tabular}




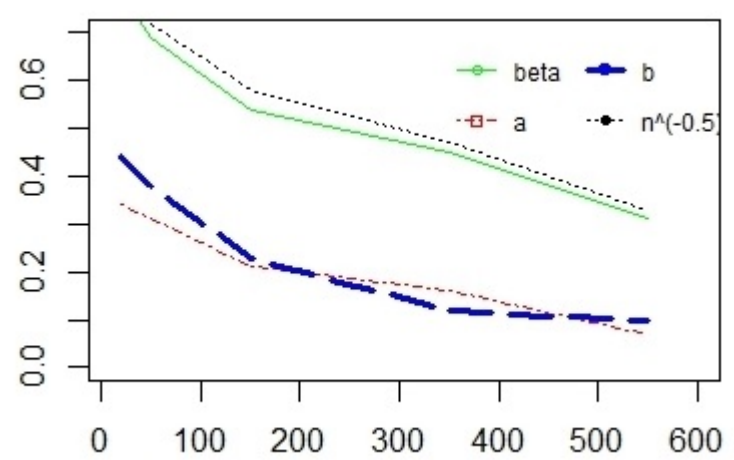

Figure 6: Simulated average absolute errors for MLEs $\widehat{\beta}, \widehat{a}, \widehat{b}$.

From the simulation results and the Figure 6, we can confirm that all estimates converge faster than $\mathrm{n}^{-0.5}$, and so our maximum likelihood estimators are $\sqrt{\mathrm{n}}$ - consistent.

\section{Maximum likelihood estimation for censored data}

\subsection{Maximum likelihood estimation}

Let $T$ be a random variable distributed according to a $\operatorname{LxIW}$ distribution with $\theta=(\beta, a, b)^{\top}$. For $i$ (individual); $T_{i}$ is the lifetime and $C_{i}$ is the censoring time, where $T_{i}$ and $C_{i}$ are independent random variables. The data consists of $n$ independent observations

$$
t_{i}=\min \left(T_{i}, C_{i}\right) \text { for } i=1, \ldots, n \text {. }
$$

The distribution of $C_{i}$ does not depend on the unknown parameters of $T_{i}$ (censoring is non-informative). Let $T_{i}$ be a random variable distributed with the vector of parameters $\theta=(\beta, a, b)^{T}$, so the likelihood function can be written as

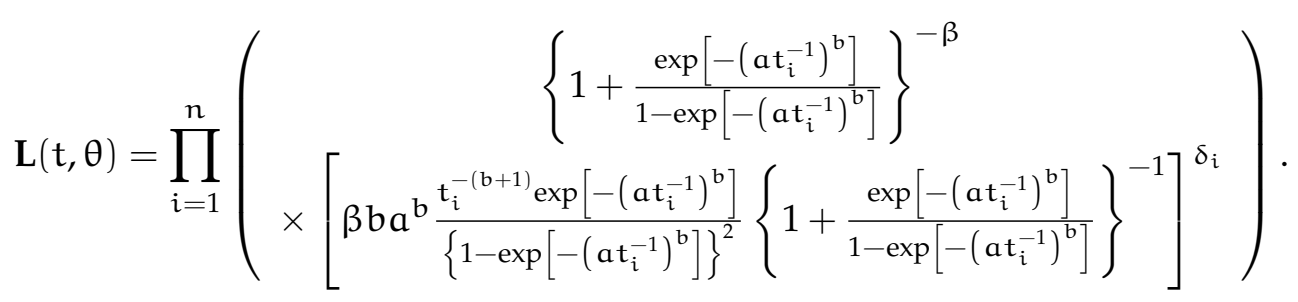

The loglikelihood function is

$$
\begin{aligned}
& l(t, \theta)=-\beta \sum_{i=1}^{n} \ln \left(1+\frac{\exp \left[-\left(a t_{i}^{-1}\right)^{b}\right]}{1-\exp \left[-\left(a t_{i}^{-1}\right)^{b}\right]}\right) \\
& +\sum_{i=1}^{n}\left[\delta_{i}\left(\begin{array}{c}
\ln \left(\beta b a^{b}\right)-(b+1) \ln \left(t_{i}\right) \\
-\left(a t_{i}^{-1}\right)^{b}-\ln \left\{1-\exp \left[-\left(a t_{i}^{-1}\right)^{b}\right]\right\}^{2} \\
-\ln \left\{1+\frac{\exp \left[-\left(a t_{i}^{-1}\right)^{b}\right]}{1-\exp \left[-\left(a t_{i}^{-1}\right)^{b}\right]}\right\}
\end{array}\right] .\right.
\end{aligned}
$$

It can be written as

$$
l(t, \theta)=r[\log (\beta)+\log (b)+b \log (a)]-(b+1) \sum_{i \in F} \ln \left(t_{i}\right)-\sum_{i \in F}\left(a t_{i}^{-1}\right)^{b}
$$




$$
-\sum_{i \in F} \ln \left(\left\{1-\exp \left[-\left(a t_{i}^{-1}\right)^{b}\right]\right\}^{2}\right)-\beta \sum_{i \in C} \ln \left\{1+\frac{\exp \left[-\left(a t_{i}^{-1}\right)^{b}\right]}{1-\exp \left[-\left(a t_{i}^{-1}\right)^{b}\right]}\right\},
$$

where $r$ is the number of failures, $F$ is the set of uncensored data and $C$ denotes censored observations. By equating the following score functions to zero, we can find our maximum likelihood estimate $\widehat{~}$ for $\theta$.

$$
\begin{aligned}
\frac{\partial l(t, \theta)}{\partial \beta}= & \frac{r}{\beta}-\sum_{i \in C} \ln \left\{1+\frac{\exp \left[-\left(a t_{i}^{-1}\right)^{b}\right]}{1-\exp \left[-\left(a t_{i}^{-1}\right)^{b}\right]}\right\}, \\
\frac{\partial l(t, \theta)}{\partial a}= & \frac{r b}{a}-\sum_{i \in F} b t_{i}\left(a t_{i}^{-1}\right)^{b-1}-2 b \sum_{i \in F} \frac{\left.t_{i} \exp \left[-\left(a t_{i}\right)\right]^{b}\right\}\left(1-\exp \left[-\left(a t_{i}\right)\right]^{b}\right)\left(a t_{i}\right)^{b-1}}{\left\{1-\exp \left[-\left(a t_{i}^{-1}\right)^{b}\right]\right\}^{2}} \\
& +\beta \sum_{i \in C} \frac{\frac{b t_{i} \exp \left[-\left(a t_{i}\right)\right]^{b}\left(a t_{i}\right)^{(b-1)}}{1-\exp \left[-\left(a t_{i}\right)\right]^{b}}-\frac{b t_{i} \exp \left[-\left(2 a t_{i}\right)\right]^{b}\left(a t_{i}\right)^{(b-1)}}{\left[1-\exp \left[-\left(a t_{i}\right)\right]^{b}\right]^{2}}}{1+\frac{\exp \left[-\left(a t_{i}^{-1}\right)^{b}\right]}{1-\exp \left[-\left(a t_{i}^{-1}\right)^{b}\right]}} \\
\frac{\partial l(t, \theta)}{\partial b}= & \frac{r}{b}+r \log (a)-\sum_{i \in F} \ln \left(t_{i}\right)-\sum_{i \in F} \ln \left(a t_{i}^{-1}\right)\left(a t_{i}^{-1}\right)^{b} \\
& -\sum_{i \in F} \frac{2 \exp \left[-\left(a t_{i}\right)^{b}\right]\left\{1-\exp \left[-\left(a t_{i}\right)^{b}\right]\right\}\left(a t_{i}\right)^{b} \ln \left(a t_{i}\right)}{\left\{1-\exp \left[-\left(a t_{i}^{-1}\right)^{b}\right]\right\}^{b}} \\
& -\beta \sum_{i \in C} \frac{-\frac{\exp \left[-\left(a t_{i}\right)^{b}\right]\left(a t_{i}\right)^{b} \ln \left(a t_{i}\right)}{1-\exp \left[-\left(a t_{i}\right)\right]^{b}}-\frac{\exp \left[-2\left(a t_{i}\right)^{b}\right](a t)^{b} \ln \left(a t_{i}\right)}{\left\{1-\exp \left[-\left(a t_{i}\right)^{b}\right]\right\}^{2}}}{1+\frac{\exp \left[-\left(a t_{i}^{-1}\right)^{b}\right]}{1-\exp \left[-\left(a t_{i}^{-1}\right)^{b}\right]}}
\end{aligned}
$$

Numerical methods, such as the Monte Carlo method, Barzilai-Borwein (BB) algorithm, Newoton Raphson algorithm or other such algorithm can be used to solve the system (quite complicated) of score functions.

\subsection{Simulations: case of censored data}

We consider the LxIW model. The data were simulated $N=10,000$ times with sample sizes $n=30, n=$ $100, n=150$ and $n=300$ and parameter values $\beta=1.6, a=2.5, b=0.7$. The averages of the simulated values of the maximum likelihood estimates $\widehat{\beta}, \widehat{a}, \widehat{b}$, and their MSEs are calculated and presented in Table 6 . From Table 6 , we can notice that the mean squared errors are very small, which confirms the convergence of the maximum likelihood estimators.

Table 6: Maximum likelihood estimates $(\widehat{\beta}, \widehat{a}, \widehat{b})$ of the parameters and their MSEs (censored data).

\begin{tabular}{|c|c|c|c|c|}
\hline $\mathrm{N}=10000$ & $\mathrm{n}=30$ & $\mathrm{n}=100$ & $\mathrm{n}=150$ & $\mathrm{n}=300$ \\
\hline$\widehat{\beta}$ & 1.7246 & 1.7198 & 1.6428 & 1.6201 \\
$\mathrm{MSE}$ & 0.04981 & 0.00314 & 0.002287 & $3.27 .10^{-4}$ \\
\hline$\widehat{\mathrm{a}}$ & 2.6214 & 2.6195 & 2.5512 & 2.5121 \\
$\mathrm{MSE}$ & 0.04857 & 0.02198 & 0.002491 & 0.00019 \\
\hline$\widehat{\mathrm{b}}$ & 0.8469 & 0.8103 & 0.7438 & 0.7211 \\
$\mathrm{MSE}$ & 0.0020 & 0.0004 & 0.0003 & 0.00018 \\
\hline
\end{tabular}

From simulation results and Figure 7 , we note that all estimates converge faster than $n^{-0.5}$, which confirm the fact that the maximum likelihood estimates are $\sqrt{n}$-consistent. 


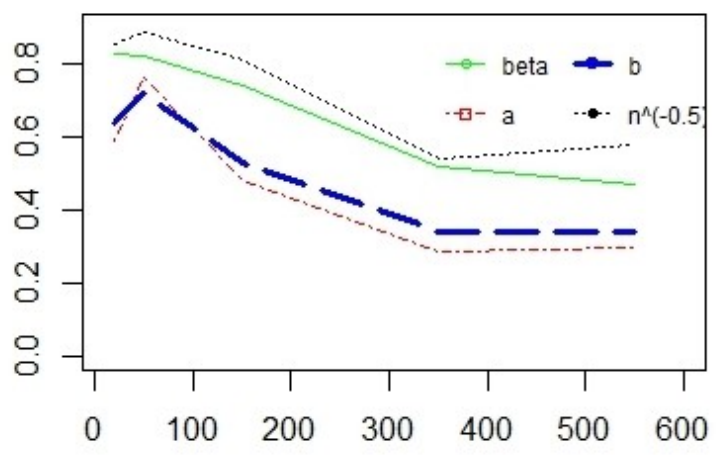

Figure 7: Simulated average absolute errors for MLEs $\widehat{\beta}, \widehat{a}, \widehat{b}$.

\section{Goodness-of-fit test}

Checking whether a series of observations can be modeled by a probability distribution is and will remain one of the most important problems in statistical analysis. To validate the choice of a model, we use what we call the goodness-of-fit test statistic.

\subsection{Nikulin-Rao-Robson test}

Different techniques are used to verify the adequacy of mathematical models to observed data. Since the middle of the last century, researchers began to propose new goodness-of-fit tests. For the complete data, Nikulin [28] and Rao and Robson [30] separately proposed a statistic known today as the N.R.R statistic (Nikulin-Rao-Robson). This statistical test, is a natural modification of the Pearson statistic. To test the hypothesis

$$
H_{0}: P\left\{T_{i} \leqslant t\right\}=F(t, \theta), \quad t \in \mathbb{R}, \quad \theta=\left(\theta_{1}, \theta_{2}, \ldots, \theta_{s}\right)^{\top},
$$

where $\theta$ represents the vector of unknown parameters of a parametric family $F(t, \theta)$, Nikulin [28] and Rao and Robson [30] proposed the N.R.R statistic $Y^{2}$ defined as follows:

Observations $T_{1}, T_{2}, \ldots, T_{n}$ are grouped in $r$ mutually disjoint subintervals $\mathbf{I}_{1}, \mathbf{I}_{2}, \ldots, \mathbf{I}_{r}$

$$
\left.\left.\mathbf{I}_{\mathbf{j}}=\right] \mathrm{a}_{\mathbf{j}-1}, \mathbf{a}_{\mathbf{j}}\right],
$$

where $j=1,2, \ldots, r$. The limits $a_{j}$ of the intervals $\mathbf{I}_{j}$ are obtained such that

$$
\left.p_{j}(\theta)\right|_{(j=1,2, \ldots, r)}=\int_{a_{j-1}}^{a_{j}} f(t, \theta) d t,
$$

so

$$
\left.a_{j}\right|_{(j=1, \ldots, r-1)}=F^{-1}\left(\frac{j}{r}\right) \text {. }
$$

If $v_{j}=\left(v_{1}, v_{2}, \ldots, v_{r}\right)^{\top}$ is the vector of frequencies obtained by the grouping of data in these $\mathbf{I}_{j}$ intervals

$$
v_{j}=\left.\sum_{i=1}^{n} 1_{\left\{t_{i} \in \mathbf{I}_{j}\right\}}\right|_{(j=1, \ldots, r)} .
$$

The N.R.R statistic is given by

$$
Y^{2}\left(\widehat{\theta}_{n}\right)=X_{n}^{2}\left(\widehat{\theta}_{n}\right)+\frac{1}{n} \mathbf{L}^{\top}\left(\widehat{\theta}_{n}\right)\left(\mathbf{I}\left(\widehat{\theta}_{n}\right)-\mathbf{J}\left(\widehat{\theta}_{n}\right)\right)^{-1} \mathbf{L}\left(\widehat{\theta}_{n}\right)
$$

where

$$
X_{n}^{2}\left(\widehat{\theta}_{n}\right)=\left(\frac{v_{1}-n p_{1}(\theta)}{\sqrt{n p_{1}(\theta)}}, \frac{v_{2}-n p_{2}(\theta)}{\sqrt{n p_{2}(\text { theta })}}, \ldots, \frac{v_{r}-n p_{r}(\theta)}{\sqrt{n p_{r}(\theta)}}\right)^{\top}
$$


and $\mathbf{J}(\theta)$ is the information matrix for the grouped data defined by

$$
\mathbf{J}(\theta)=B(\theta)^{\top} B(\theta),
$$

with

and

$$
\left.\mathrm{B}(\theta)\right|_{(i=1,2, \ldots, \mathrm{r} \text { and } k=1, \ldots, \mathrm{s})}=\left[\frac{1}{\sqrt{p}_{\mathrm{i}}} \frac{\partial \mathrm{p}_{\mathrm{i}}\left(^{\prime}\right)}{\partial \mu}\right]_{\mathrm{r} \times \mathrm{s}},
$$

$$
\mathbf{L}(\theta)=\left(\mathbf{L}_{1}(\text { theta }), \ldots, \mathbf{L}_{s}(\theta)\right)^{\top} \quad \mathbf{L}_{k}(\theta)=\sum_{i=1}^{r} \frac{v_{i}}{p_{i}} \frac{\partial}{\partial \theta_{k}} p_{i}(\theta),
$$

where in $\mathbf{I}\left(\widehat{\theta_{n}}\right)$ represented above, the estimated Fisher information matrix and $\widehat{\theta_{n}}$ is the maximum likelihood estimator of the parameter vector. The $Y^{2}$ statistic follows a chi-square distribution with $(r-1)$ degrees of freedom.

\subsection{N.R.R statistic for the LxIW model}

To verify if a sample $T=\left(T_{1}, T_{2}, \ldots, T_{n}\right)^{T}$ is distributed according to the LxIW model, $P\left\{T_{i} \leqslant t\right\}=F_{L x I W}(t, \theta)$; with unknown parameters $\theta=(\beta, a, b)^{\top}$, a chi-square goodness-of-fit test is constructed by fitting the N.R.R statistic developed in the previous section. The maximum likelihood estimators $\widehat{\theta_{n}}$ of the unknown parameters of the LxIW distribution are computed on the initial data. The statistic $Y^{2}$ does not depend on the parameters, we can therefore use the estimated Fisher information matrix $\mathbf{I}\left(\widehat{\theta_{n}}\right)$.

\subsection{Simulation studies (N.R.R statistics $Y^{2}$ )}

To test the null hypothesis $\mathrm{H}_{0}$ that the sample arose from the LxIW model, we calculate $\mathrm{Y}^{2}$ the N.R.R statistic using 10,000 simulated samples with sizes

$$
n=30, n=50, n=100, n=250 \text {, and } n=500 .
$$

For different theoretical levels $(\epsilon=0.02,0.05,0.01,0.1)$, we calculate the average of the non-rejection numbers of the null hypothesis, when $Y^{2} \leqslant \chi_{\epsilon}^{2}(r-1)$. Then, we present the results of the corresponding empirical and theoretical levels in Table 7. As can be seen, the values of the empirical levels calculated are very close to those of their corresponding theoretical levels. Thus, we conclude that the proposed test accepts the hypothesis that the samples arose from the LxIW distribution.

Table 7: Empirical levels and corresponding theoretical levels $(\epsilon=0.02,0.05,0.01,0.1)$.

\begin{tabular}{|c|c|c|c|c|}
\hline $\mathrm{N}=10000$ & $\epsilon=0.02$ & $\epsilon=0.05$ & $\epsilon=0.01$ & $\epsilon=0.1$ \\
\hline $\mathrm{n}=30$ & 0.9834 & 0.9541 & 0.9944 & 0.9050 \\
\hline $\mathrm{n}=50$ & 0.9830 & 0.9532 & 0.9930 & 0.9028 \\
\hline $\mathrm{n}=100$ & 0.9819 & 0.9520 & 0.9921 & 0.9018 \\
\hline $\mathrm{n}=250$ & 0.9804 & 0.9508 & 0.9907 & 0.9009 \\
\hline $\mathrm{n}=500$ & 0.9802 & 0.9502 & 0.9903 & 0.9001 \\
\hline
\end{tabular}

\subsection{Simulated distribution of $\mathrm{Y}^{2}$ statistic for LxIW model}

The $Y^{2}$ statistic follows in the limit a chi-squared distribution with $k=r-1$ degrees of freedom. For demonstrating this fact, we compute $\mathrm{N}=10,000$ times, the simulated distribution of $\mathrm{Y}^{2}(\widehat{\theta})$ under the null hypothesis $\mathrm{H}_{0}$ with different values of parameters, and $r=12$ intervals, versus the chi-squared distribution with $k=11$ degree of freedom. Their histograms are represented in Figure 8 versus the chi-squared distribution with 11 degrees of freedom.

From Figure 8, we observe that the statistical distribution of $Y^{2}$ with different values of parameters and different numbers $k$ of grouping cells; for different number of equiprobable grouping intervals and different value of parameters; in the limit follows a chi-squared with $k$ degrees of freedom within the statistical errors of simulation. We can say that the limiting distribution of the generalized chi-squared $Y^{2}$ statistic for LxIW model is distribution free. 

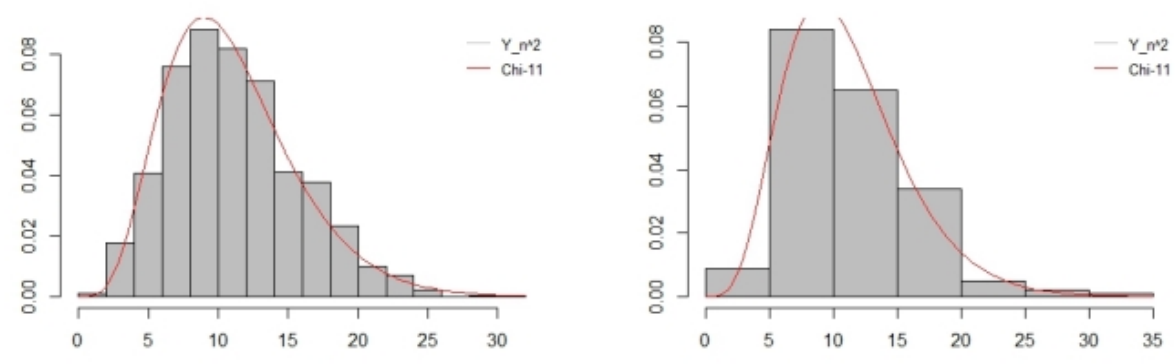

(a) $\widehat{\theta}=(\widehat{\beta}=1.5, \widehat{a}=1.5, \widehat{b}=2.5)$

(b) $\widehat{\theta}=(\widehat{\beta}=0.5, \widehat{a}=1.2, \widehat{b}=2.5)$
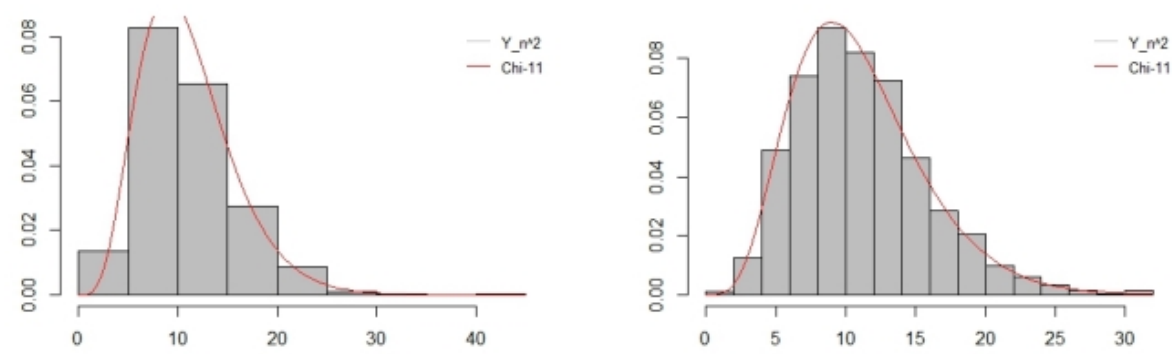

(c) $\widehat{\theta}=(\widehat{\beta}=0.5, \widehat{a}=0.5, \widehat{b}=2.5)$

(d) $\widehat{\theta}=(\widehat{\beta}=0.5, \widehat{a}=0.5, \widehat{b}=1.5)$

Figure 8: Simulated distribution of the $\mathrm{Y}^{2}$ statistic under the null hypothesis $\mathrm{H}_{0}$, with different parameters of $\widehat{\theta}$ versus the chi-squared distribution with 11 degrees of freedom, with $n=150, N=10000$.

\subsection{Applications to real data}

\subsubsection{Breaking stress of carbon fibres (in Gba) data}

To test the null hypothesis $\mathrm{H}_{0}$ that these data are from a LxIW distribution, we use the N.R.R statistic mentioned previously. Using the R software and the BB algorithm (Ravi [32]), we compute the maximum likelihood estimates (MLE) $\widehat{\beta}=7.1524, \widehat{a}=1.5607, \widehat{b}=0.51149$. The estimated Fisher information matrix is then

$$
\mathbf{I}(\widehat{\theta})=\left(\begin{array}{ccc}
0.095416 & 0.248130 & 9.2487992 \\
& 61.589475 & 214.921433 \\
& & 97.51899
\end{array}\right) .
$$

We then deduce the value of $Y^{2}=16.30498$. The critical value is

$$
\chi_{0.05}^{2}(10-1)=16.91898
$$

then, the value of N.R.R $Y^{2}$ statistic is less than the critical value, which shows that the data arose from a LxIW model.

\subsubsection{Strengths of $1.5 \mathrm{~cm}$ glass fibres}

Assuming that the Strengths of $1.5 \mathrm{~cm}$ glass fibres data can be fitted by our LxIW model, we find (using the BB algorithm) the MLE's of the $\theta$ vector of parameters as:

$$
\widehat{\theta}=(\widehat{\beta}, \widehat{a}, \widehat{b})^{\top}=(6.3254,0.95277,1.0243)^{\top}
$$


Using the $\widehat{\theta}$ values, the estimated Fisher information matrix is can be writing as:

$$
\mathbf{I}(\widehat{\theta})=\left(\begin{array}{ccc}
0.524876 & 0.1283345 & 4.128756 \\
& 18.54996 & 64.12587 \\
& & 247.99912
\end{array}\right) .
$$

After calculation, we have the value of N.R.R test statistic and the critical value as: $Y^{2}=11.6844$ and $\chi_{0.05}^{2}(7-1)=$ 12.59159 , respectively. We maintain that data of $1.5 \mathrm{~cm}$ glass fibres fits well to our LxIW model.

\section{Goodness-of-fit test for right censored data}

Habib and Thomas [18] considered the natural modifications of the N.R.R statistic. These tests are based on the differences between two probability estimators, one based on the Kaplan-Meier estimator, the other based on the maximum likelihood estimators of the unknown parameters of the cumulative distribution function of the Kaplan-Meier estimator. Bagdonavicius and Nikulin [5] and Bagdonavicius et al. [3], proposed a modification of the N.R.R statistic that takes into account random right censoring, this statistic based on the maximum likelihood estimators on the initial data also follows a limiting Chi-square distribution. For more details on the construction of these statistics, we can see Balakrishnan et al. [6]. These techniques were used to test the fit of the observations to the generalized inverse Weibull model (Goual and Seddik-Ameur [15]), the distribution of Birbaurm Saunders (Couallier et al. [10]), the Kumaraswamy generalized inverse Weibull distribution (Goual and Seddik-Ameur [16]), Bertholon model (Chouia and Seddik-Ameur [8]) and others. In this section we develop the approach proposed by Bagdonavicius and Nikulin [5] and Bagdonavicius et al. [3]; to confirm the adequacy of LxIW model when the parameters are unknown and data are censored. Let us consider the composite hypothesis

$$
H_{0}: F(t) \in F_{0}=\left\{F_{0}(t, \theta), t \in R^{1}, \theta \in \Theta \subset R^{s}\right\},
$$

where

$$
\theta=\left(\theta_{1}, \ldots, \theta_{s}\right)^{\top} \in \Theta \subset R^{s}
$$

is an unknown s-dimensional parameter and $F_{0}$ is a differentiable and specified completely cdf with support $(0, \infty)$. Let us consider a finite time interval only say $[0, \tau]$, where $\tau$ is the maximum time of the study, and divide it into $k>s$ smaller intervals $\mathbf{I}_{j}=\left(\boldsymbol{a}_{j-1}, \boldsymbol{a}_{\mathbf{j}}\right]$, where

$$
0=<a_{0}<a_{1}<\cdots<a_{k-1}<a_{k}=+\infty .
$$

In this case the estimated $\widehat{a}_{j}$ is given by

$$
\widehat{a}_{j}=\Lambda^{-1}\left(\left(E_{j}-\sum_{l=1}^{i-1} \Lambda\left(T_{(l)}, \widehat{\theta}\right)\right) /(n-i+1), \widehat{\theta}\right), \quad \widehat{a_{k}}=T_{(n)}, j=1, \ldots, k,
$$

where $\widehat{\theta}$ is the maximum likelihood estimator of the parameter $\theta, \Lambda^{-1}$ is the inverse of cumulative hazard function $\Lambda, T_{(i)}$ is the $i^{\text {th }}$ element in the ordered statistics $\left(T_{(1),,}, T_{(n)}\right)$ and

$$
E_{j}=(n-i+1) \wedge\left(\widehat{a_{j}}, \widehat{\theta}\right)+\sum_{l=1}^{i-1} \Lambda\left(T_{(l)}, \widehat{\theta}\right),
$$

and $a_{j}$ are random data functions such as the $k$ intervals chosen with equal expected numbers of failures $e_{j}$. Usually in real application we fix k. Bagdonavicius et al. [2] and Greenwood and Nikulin [17] give some recommendations for the choice of intervals. The test is based on the vector

$$
\mathbf{Z}=\left(\mathbf{Z}_{1}, \ldots, \mathbf{Z}_{k}\right)^{\top}, \quad \mathbf{Z}_{j}=\frac{1}{\sqrt{n}}\left(\mathbf{U}_{j}-e_{j}\right), \quad j=1,2, \ldots, k,
$$

where $\mathbf{U}_{j}$ represent the numbers of observed failures in these intervals. The test for hypothesis $\mathrm{H}_{0}$ can be based on the statistic

$$
Y_{n}^{2}=\mathbf{Z}^{\top} \hat{\Sigma}^{-1} \mathbf{Z}
$$

where

$$
\widehat{\Sigma}^{-1}=\widehat{\mathbf{A}}^{-1}+\widehat{\mathbf{C}}^{-1} \widehat{\mathbf{A}}^{\top} \widehat{\mathbf{G}}^{-1} \widehat{\mathbf{C}} \widehat{\mathbf{A}}^{-1}
$$


and

$$
\widehat{\mathbf{G}}=\widehat{i}-\widehat{\mathbf{C}} \widehat{\mathbf{A}}^{-1} \widehat{\mathbf{C}}^{\mathrm{T}} .
$$

The test statistic can be written in the following form

$$
Y_{n}^{2}=\sum_{j=1}^{k} \frac{1}{\mathbf{U}_{j}}\left(\mathbf{U}_{j}-e_{j}\right)^{2}+\mathbf{Q},
$$

where

$$
\begin{aligned}
\widehat{\mathbf{A}}_{j} & =n^{-1} \mathbf{U}_{j}, \\
\widehat{\mathbf{G}} & =\left[\widehat{\mathrm{g}}_{l l^{\prime}}\right]_{s \times s}, \\
\mathbf{U}_{j} & =\sum_{i: X_{i} \in \mathbf{I}_{j}} \delta_{i}, \\
\mathbf{Q} & =\widehat{\mathbf{W}}^{\top} \widehat{\mathbf{G}}^{-1} \widehat{\mathbf{W}}, \\
\widehat{\mathbf{C}}_{\mathrm{lj}} & =\frac{1}{n} \sum_{i: X_{i} \in \mathbf{I}_{j}} \delta_{i} \frac{\partial}{\partial \theta} \ln \left[\lambda_{i}\left(t_{i}, \widehat{\theta}\right)\right], \\
\widehat{\mathbf{W}}_{l} & =\sum_{j=1}^{k} \widehat{\mathbf{C}}_{l j} \widehat{\mathbf{A}}_{j}^{-1} \mathbf{Z}_{j}, \quad l, l^{\prime}=1, \ldots, s, \\
\widehat{\mathbf{W}} & =\left(\widehat{\mathbf{W}}_{1}, \widehat{\mathbf{W}}_{2}, \ldots, \widehat{\mathbf{W}}_{s}\right)^{\top}, \\
\widehat{i}_{l l^{\prime}} & =n^{-1} \sum_{i=1}^{n} \delta_{i} \frac{\partial}{\partial \theta_{l}} \ln \left[\lambda_{i}\left(t_{i}, \widehat{\theta}\right)\right] \frac{\partial}{\partial \theta_{l^{\prime}}} \ln \left[\lambda_{i}\left(t_{i}, \widehat{\theta}\right)\right],
\end{aligned}
$$

and

$$
\widehat{\mathrm{g}}_{l l^{\prime}}=\widehat{i}_{l l^{\prime}}-\sum_{j=1}^{k} \widehat{\mathbf{C}}_{l j} \widehat{\mathbf{C}}_{l^{\prime} j} \hat{A}_{j}^{-1}, \widehat{\mathbf{C}}_{l j}=\frac{1}{n} \sum_{i: X_{i} \in \mathbf{I}_{j}} \delta_{i} \frac{\partial}{\partial \theta} \ln \lambda_{i}\left(t_{i}, \widehat{\theta}\right) .
$$

The elements of the matrices $\widehat{\mathbf{W}}$ and $\widehat{\mathbf{I}}$ are given in the Appendix. The limiting distribution of the statistic $Y_{\mathrm{n}}^{2}$ is chi-square with $r=\operatorname{rank}(\Sigma)=\operatorname{tr}\left(\Sigma^{-1} \Sigma\right)$ degrees of freedom. If $\mathbf{G}$ is non-degenerate then $r=k$. The hypothesis is rejected with approximate significance level $\epsilon$ if $Y_{n}^{2}>\chi_{\epsilon}^{2}(r)$ where $\chi_{\epsilon}^{2}(r)$ is the $1-\epsilon$ quantile of chi-square with $r$ degrees of freedom. For more details, see Bagdonavicius and Nikulin [5] and Bagdonavicius et al. [3].

\subsection{Validation of LxIW model in case of censored data}

In this section, we study the validity of the LxIW model, by a goodness-of-fit test based on $Y_{n}^{2}$, the modified N.R.R statistic presented in the previous section. Suppose $\mathrm{H}_{0}$ is $\mathrm{T}_{i}$ follows LxIW distribution. The survival function is:

$$
S(t, \theta)=1-F(t ; \beta, a, b)=\left\{1+\frac{\exp \left[-\left(a t^{-1}\right)^{b}\right]}{1-\exp \left[-\left(a t^{-1}\right)^{b}\right]}\right\}^{-\beta},
$$

when the baseline distribution is a LxIW model, the choice of $\widehat{a}_{j}$, is given by

and

$$
\Lambda_{\mathrm{LxIW}}(\mathrm{t}, \theta)=-\ln S(\mathrm{t}, \theta)=\beta \ln \left\{1+\frac{\exp \left[-\left(\mathrm{at}^{-1}\right)^{\mathrm{b}}\right]}{1-\exp \left[-\left(\mathrm{at}^{-1}\right)^{\mathrm{b}}\right]}\right\}
$$

$$
\begin{aligned}
t & =a\left(\ln \left\{[\exp (y / \beta)-1]^{-1}+1\right\}\right)^{\frac{-1}{b}}, \\
\left.\widehat{a}_{j}\right|_{(j=1, \ldots, k-1)} & =a\left[\ln \left(\left\{\exp \left[\frac{E_{j}-\sum_{l=1}^{i-1} \wedge\left(x_{(l)}, \widehat{\theta}\right)}{\beta(n-i+1)}\right]-1\right\}^{-1}+1\right)\right]^{-\frac{1}{b}},
\end{aligned}
$$




$$
\widehat{\mathrm{a}}_{\mathrm{k}}=\mathrm{t}_{(\mathrm{n})},
$$

where

$$
E_{j}=\sum_{i: X_{i}>a_{j}}\left(\Lambda\left(a_{j} \wedge t_{i}, \widehat{\theta}\right)-\Lambda\left(a_{j-1}, \widehat{\theta}\right) \text { and } E_{k}=\sum_{i=1}^{n} \Lambda\left(t_{i}, \widehat{\theta}\right),\right.
$$

with this choice of intervals, for any $j$ we have a constant value of $e_{j}=E_{k} / k$. Intervals can be estimated by iterative method (there is no explicit form of inverse hazard function of LxIW distribution).

\subsection{Simulation study}

To test the null hypothesis $\mathrm{H}_{0}$ that a sample comes from a LxIW model, we calculate $Y_{\mathrm{n}}^{2}$ the N.R.R statistic of 10,000 simulated samples with sizes $n=30, n=100, n=200$ and $n=500$. For different significance levels $\epsilon=0.02,0.05,0.01,0.1$, we calculate the mean of the number of no rejections of the null hypothesis when $Y_{n}^{2} \leqslant \chi_{\epsilon}^{2}(r)$, then we present the results of the empirical values and the corresponding theoretical values in Table 8.

Table 8: Empirical levels and corresponding theoretical levels $(\epsilon=0.02 ; 0.05 ; 0.01 ; 0.1)$.

\begin{tabular}{|c|c|c|c|c|}
\hline $\mathrm{N}=10000$ & $\epsilon=0.02$ & $\epsilon=0.05$ & $\epsilon=0.01$ & $\epsilon=0.1$ \\
\hline $\mathrm{n}=30$ & 0.9829 & 0.9530 & 0.9920 & 0.9018 \\
\hline $\mathrm{n}=100$ & 0.9819 & 0.9513 & 0.9916 & 0.9015 \\
\hline $\mathrm{n}=200$ & 0.9804 & 0.9506 & 0.9909 & 0.9010 \\
\hline $\mathrm{n}=500$ & 0.9802 & 0.9503 & 0.9903 & 0.9899 \\
\hline
\end{tabular}

According to these results, we find that the empirical significance levels of the $Y_{n}^{2}$ statistic coincide with those corresponding to the theoretical levels of the chi-square distributions at 11 degrees of freedom. Therefore, we can say that the proposed test can properly fit censored data from the LxIW distribution.

\subsection{Application to real data}

\subsubsection{Aluminum reduction cells data}

The data of Whitmore (1983), who considered the times of failures for 20 aluminum reduction cells, and the numbers of failures in 1,000 days units are : $0.468,0.725,0.838,0.853,0.965,1.139,1.142,1.304,1.317,1.427,1.554$, $1.658,1.764,1.776,1.990,2.010,2.224,2.279^{*}, 2.244^{*}, 2.286^{*}{ }^{*}$ censored). Assuming that these data are distributed according to the LxIW distribution, the maximum likelihood estimator $\widehat{\theta}$ of the parameter vector $\theta$ is

$$
\widehat{\theta}=(\widehat{\beta}, \widehat{a}, \widehat{b})^{\top}=(1.6148,0.92145,0.84571)^{\top} .
$$

We choose $r=4$ as number of classes. The elements of the test statistic $Y_{n}^{2}$ are presented as follows:

$$
\begin{array}{ccccc}
\widehat{a_{j}} & 0.8425 & 1.2876 & 1.7904 & 2.3211 \\
\widehat{\mathbf{U}_{j}} & 3 & 4 & 7 & 6 \\
\mathrm{e}_{j} & 2.4587 & 2.4587 & 2.4587 & 2.4587 \\
\widehat{\mathrm{C}_{1 j}} & -0.84579 & 0.312845 & 0.402875 & 0.01458 \\
\widehat{\mathrm{C}_{2 j}} & 0.19547 & -0.42015 & 0.11489 & 0.249821 \\
\widehat{\mathrm{C}_{3 j}} & 0.015594 & -0.021548 & 0.64781 & 0.009521
\end{array}
$$

The estimated Fisher's matrix is given by

$$
\widehat{\mathbf{I}}=\left(\begin{array}{lll}
2.30149 & -5.28461 & 1.002184 \\
& -1.20457 & 0.449327 \\
& & 3.254419
\end{array}\right) .
$$


Then we calculate the value of the test statistic as $Y_{n}^{2}=9.1098$. The critical value is

$$
\chi_{0.05}^{2}(4)=9.4877>Y_{n}^{2},
$$

and hence we reason that the data of Aluminum reduction cells is compatible with LxIW model.

\subsubsection{Myeloid leukemia data}

We consider Freireich et al. [14] sample data from 51 patients with advanced acute myeloid leukemia reported in the International Registry of Bone Marrow Transplantation. These patients had received an autologous bone marrow transplant in which, after large doses of chemotherapy, their own marrow was reintroduced to replace their destroyed immune system. Survival time (in months) for autologous transplants is: $0.658,0.822,1.414$, 2.5, 3.322, 3.816, 4.737, 4.836* 4.934, 5.033, 5.757,5.855, 5.987, 6.151,6.217, 6.447*, 8.651, 8.717, 9.441*, 10.329, 11.48

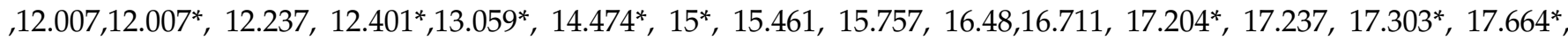
$18.092,18.092^{*}, 18.75^{*}, 20.625^{*}, 23.158,27.73^{*}, 31.184^{*}, 32.434^{*}, 35.921^{*}, 42.237^{*}, 44.638^{*}, 46.48^{*}, 47.467^{*}, 48.322^{*}$, 56.086. If the myeloid leukemia data is distributed as LxIW, the maximum likelihood estimator $\hat{\theta}$ of the parameter vector $\theta$ is then:

$$
\widehat{\theta}=(\widehat{\beta}, \widehat{a}, \widehat{b})^{\top}=(1.9588,0.8627,2.5491)^{\top} .
$$

We choose $r=7$ as number of classes. The elements of the test statistic $Y_{n}^{2}$ are :

$\begin{array}{cccccccc}\widehat{\mathrm{a}_{j}} & 4.752 & 12.157 & 14.204 & 16.912 & 22.348 & 41.007 & 56.102 \\ \widehat{\mathrm{U}_{j}} & 7 & 16 & 3 & 6 & 8 & 5 & 6 \\ \mathrm{e}_{j} & 1.9534 & 1.9534 & 1.9534 & 1.9534 & 1.9534 & 1.9534 & 1.9534 \\ \widehat{\mathrm{C}_{1 j}} & 0.3120 & 0.2837 & -0.3418 & -0.10542 & 0.3124 & 0.3954 & 0.1197 \\ \widehat{\mathrm{C}_{2 j}} & 0.6417 & 0.5921 & 0.1394 & 0.25876 & 0.4981 & -0.4258 & -0.0947 \\ \widehat{\mathrm{C}_{3 j}} & 0.08946 & -0.2430 & 0.0894 & 0.2413 & 0.2539 & 0.17843 & 0.6479\end{array}$

The Fisher's estimated matrix is:

$$
\widehat{\mathbf{I}}=\left(\begin{array}{ccc}
1.38954 & 1.922301 & 2.14587 \\
& 4.228718 & -1.28343 \\
& & -0.318211
\end{array}\right) .
$$

After computing, our test statistic is $Y_{n}^{2}=13.92441$. The critical value $\chi_{0.05}^{2}(7)=14.06714>Y_{n}^{2}=13.92441$. Hence one can state that LxIW distribution fits well to the myeloid leukemia data.

\section{Conclusions}

In this work, we proposed a new generalized distribution called the Lomax Inverse Weibull distribution (LxIW), and studied and explored various mathematical and statistical properties associated with this distribution. Using the modified goodness of fit test statistic $Y_{n}^{2}$ proposed by Bagdonavicius and Nikulin [5], we have validated our new model in the presence of right censored. We have done a large study by simulation to demonstrate the flexibility of the proposed distribution in survival analysis and reliability, and the efficiency of the studied estimation method. Some real data of both complete and censored cases were analyzed to confirm the suitability of the proposed test and the suitatbility of this distribution to model certain life data.

\section{Appendix}

The components of $\widehat{\mathbf{W}}$ and the information matrix $\widehat{\mathbf{I}}$ are required for the test statistic $Y_{n}^{2}$. 


\section{Calculation of the matrix $\widehat{W}$}

The elements of the estimated matrix $\widehat{\mathbf{W}}$ defined by

$$
\widehat{\mathbf{W}}_{\mathbf{l}}=\left.\sum_{j=1}^{\mathrm{k}} \widehat{\mathbf{C}}_{\mathrm{lj}} \widehat{\mathbf{A}}_{j}^{-1} \mathbf{Z}_{\mathbf{j}}\right|_{(\mathbf{l}=1,2,3 \text { and } \mathrm{j}=1, \ldots, \mathrm{k})},
$$

are obtained as follow

$$
\begin{aligned}
\widehat{\mathbf{C}_{l j}}= & \frac{1}{n} \sum_{i . t_{i} \in \mathbf{I}_{j}} \delta_{i} \frac{\partial}{\partial \cdot} \ln \lambda\left(t_{i}, \widehat{r}\right), \\
\ln \lambda(t, \widehat{r})= & \ln \left(f_{i b a^{b}}\right)-(b+1) \ln \left(t_{i}\right)-\left(a t_{i}^{-1}\right)^{b} \\
& -\ln \left\{1-\exp \left[-\left(a t_{i}^{-1}\right)^{b}\right]\right\}^{2}-\ln \left\{1+\frac{\exp \left[-\left(a t_{i}^{-1}\right)^{b}\right]}{1-\exp \left[-\left(a t_{i}^{-1}\right)^{b}\right]}\right\} .
\end{aligned}
$$

The expressions of the elements of the matrix $\widehat{\mathrm{C}_{\mathrm{lj}}}$ are given as follows

$$
\begin{aligned}
& \widehat{\mathbf{C}_{1 j}}=\frac{1}{n} \sum_{i . t_{i} \in \mathbf{I}_{j}} \delta_{i}\left[\ln \left\{1+\frac{\exp \left[-\left(a t_{i}^{-1}\right)^{b}\right]}{1-\exp \left[-\left(a t_{i}^{-1}\right)^{b}\right]}\right\}\right] \\
& \widehat{\mathbf{C}_{2 j}}=\frac{1}{n} \sum_{i . t_{i} \in \mathbf{I}_{j}} \delta_{i}\left[\begin{array}{c}
\frac{b}{a}-b t_{i}\left(a t_{i}^{-1}\right)^{b-1} \\
-2 b \frac{\left.t_{t_{i}} \exp \left[-\left(a t_{i}\right)\right]^{b}\right\}\left(1-\exp \left[-\left(a t_{i}\right)\right]^{b}\right)\left(a t_{i}\right)^{(b-1)}}{\left\{1-\exp \left[-\left(a t_{i}^{-1}\right)^{b}\right]\right\}^{2}}
\end{array}\right] \text {, } \\
& \widehat{\mathbf{C}_{3 j}}=\frac{1}{n} \sum_{i . t_{i} \in \mathbf{I}_{j}} \delta_{i}\left[\begin{array}{c}
\frac{1}{b}+\log (a)-\ln \left(t_{i}\right)-\ln \left(a t_{i}^{-1}\right)\left(a t_{i}^{-1}\right)^{b} \\
-\frac{2 \exp \left[-\left(a t_{i}\right)^{b}\right]\left\{1-\exp \left[-\left(a t_{i}\right)^{b}\right]\right\}\left(a_{i}\right)^{b} \ln \left(a t_{i}\right)}{\left\{1-\exp \left[-\left(a t_{i}^{-1}\right)^{b}\right]\right\}^{2}}
\end{array}\right] \text {. }
\end{aligned}
$$

\section{Calculation of the matrix $\widehat{\mathbf{I}}$}

The formulas of the elements of the estimated Fisher's information matrix $\widehat{\mathbf{I}}=\left(\hat{\mathfrak{i}}_{l l^{\prime}}\right)_{3 \times 3}$ is

$$
\widehat{i_{l l^{\prime}}}=\frac{1}{n} \sum_{i . t_{i} \in \mathbf{I}_{j}} \delta_{i} \frac{\partial \ln \lambda\left(t_{i}, \widehat{ }\right)}{\partial{ }^{\prime}} \frac{\partial \ln \lambda\left(t_{i}, \widehat{{ }^{\prime}}\right)}{\partial^{\prime} \iota^{\prime}}
$$

In our case we have:

$$
\begin{aligned}
& \widehat{i_{11}}=\frac{1}{n} \sum_{i . t_{i} \in \mathbf{I}_{j}} \delta_{i}\left[\ln \left\{1+\frac{\exp \left[-\left(a t_{i}^{-1}\right)^{b}\right]}{1-\exp \left[-\left(a t_{i}^{-1}\right)^{b}\right]}\right\}\right]^{2}, \\
& \widehat{i_{12}}=\frac{1}{n} \sum_{i . t_{i} \in \mathbf{I}_{j}} \delta_{i i}\left(\begin{array}{l}
\ln \left\{1+\frac{\exp \left[-\left(a t_{i}^{-1}\right)^{b}\right]}{1-\exp \left[-\left(a t_{i}^{-1}\right)^{b}\right]}\right\} \\
\left.\left.\frac{b}{a}-b t_{i}\left(a t_{i}^{-1}\right)^{b-1}-2 b \frac{\left.t_{i} \exp \left[-\left(a t_{i}\right)\right]^{b}\right\}\left(1-\exp \left[-\left(a t_{i}\right)\right]^{b}\right)\left(a t_{i}\right)^{(b-1)}}{\left\{1-\exp \left[-\left(a t_{i}^{-1}\right)^{b}\right]\right\}^{2}}\right\}\right), \\
\widehat{i_{13}}=\frac{1}{n} \sum_{i . t_{i} \in \mathbf{I}_{j}} \delta_{i}\left(1+\frac{\exp \left[-\left(a t_{i}^{-1}\right)^{b}\right]}{1-\exp \left[-\left(a t_{i}^{-1}\right)^{b}\right]}\right\} \\
\times\left\{\begin{array}{c}
\frac{1}{b}+\log (a)-\ln \left(t_{i}\right)-\ln \left(a t_{i}^{-1}\right)\left(a t_{i}^{-1}\right)^{b} \\
\left.-\frac{2 \exp \left[-\left(a t_{i}\right)^{b}\right]\left\{1-\exp \left[-\left(a t_{i}\right)^{b}\right]\right\}\left(a t_{i}\right)^{b} \ln \left(a t_{i}\right)}{\left\{1-\exp \left[-\left(a t_{i}^{-1}\right)^{b}\right]\right\}^{2}}\right\}
\end{array}\right),
\end{array}\right.
\end{aligned}
$$




$$
\begin{aligned}
& \widehat{i_{22}}=\frac{1}{n} \sum_{i . t_{i} \in \mathbf{I}_{j}} \delta_{i}\left[\begin{array}{c}
\frac{b}{a}-b t_{i}\left(a t_{i}^{-1}\right)^{b-1} \\
-2 b \frac{\left.t_{i} \exp \left[-\left(a t_{i}\right)\right]^{b}\right\}\left(1-\exp \left[-\left(a t_{i}\right)\right]^{b}\right)\left(a t_{i}\right)^{(b-1)}}{\left\{1-\exp \left[-\left(a t_{i}^{-1}\right)^{b}\right]\right\}^{2}}
\end{array}\right]^{2}, \\
& \widehat{i_{23}}=\frac{1}{n} \sum_{i . t_{i} \in \mathbf{I}_{j}} \delta_{i}\left\{\frac{1}{\mathbf{f i}^{2}}\left[\begin{array}{c}
\frac{b}{a}-b t_{i}\left(a t_{i}^{-1}\right)^{b-1}-2 b \frac{\left.t_{i} \exp \left[-\left(a t_{i}\right)\right]^{b}\right\}\left(1-\exp \left[-\left(a t_{i}\right)\right]^{b}\right)\left(a t_{i}\right)^{(b-1)}}{\left\{1-\exp \left[-\left(a t_{i}^{-1}\right)^{b}\right]\right\}^{2}} \\
-\frac{1}{b}+\log (a)-\ln \left(t_{i}\right)-\ln \left(a t_{i}^{-1}\right)\left(a t_{i}^{-1}\right)^{b} \\
-\frac{2 \exp \left[-\left(a t_{i}\right)^{b}\right]\left\{1-\exp \left[-\left(a t_{i}\right)^{b}\right]\right\}\left(a t_{i}\right)^{b} \ln \left(a t_{i}\right)}{\left\{1-\exp \left[-\left(a t_{i}^{-1}\right)^{b}\right]\right\}^{2}}
\end{array}\right],\right.
\end{aligned}
$$

and

$$
\widehat{i_{33}}=\frac{1}{n} \sum_{i . t_{i} \in \mathbf{I}_{j}} \delta_{i}\left[\begin{array}{c}
\frac{1}{b}+\log (a)-\ln \left(t_{i}\right)-\ln \left(a t_{i}^{-1}\right)\left(a t_{i}^{-1}\right)^{b} \\
-\frac{2 \exp \left[-\left(a t_{i}\right)^{b}\right]\left\{1-\exp \left[-\left(a t_{i}\right)^{b}\right]\right\}\left(a t_{i}\right)^{b} \ln \left(a t_{i}\right)}{\left\{1-\exp \left[-\left(a t_{i}^{-1}\right)^{b}\right]\right\}^{2}}
\end{array}\right]^{2} .
$$

\section{References}

[1] A. Z. Afify, H. M. Yousof, G. M. Cordeiro, E. M. M. Ortega, Z. M. Nofal, The Weibull Fréchet distribution and its applications, J. Appl. Stat., 43 (2016), 2608-2626 3.2

[2] V. B. Bagdonavicius, J. Kruopis, M. Nikulin, Nonparametric tests for Censored Data, ISTE and J. Wiley, London, (2010). 6

[3] V. B. Bagdonavicius, R. J. Levuliene, M. S. Nikulin, Chi-squared goodness-of-fit tests for parametric accelerated failure time models, Comm. Statist. Theory Methods, 42 (2013), 2768-2785. 6

[4] V. B. Bagdonavicius, M. S. Nikulin, Chi-squared Goodness-of-fit Test for Right Censored Data, Int. J. Appl. Math. Stat., 24 (2011), 30-50.

[5] V. Bagdonavicius, M. Nikulin, Chi-squared tests for general composite hypotheses from censored samples, C. R. Math. Acad. Sci. Paris, 349 (2011), 219-223. 6, 7

[6] N. Balakrishnan, V. Voinov, M. S. Nikulin, Chi-Squared Goodness of Fit Tests with Applications, Academic Press, New York, (2013). 6

[7] M. Bourguignon, R. B. Silva, G. M. Cordeiro, The Weibull-G family of probability distributions, J. Data Sci., 12 (2014), 53-68.

[8] S. Chouia, N. Seddik-Ameur, A modified chi-square test for Bertholon model with censored data, Comm. Statist. Simulation Comput., 46 (2017), 593-602. 6

[9] G. M. Cordeiro, H. M. Yousof, T. G. Ramires, E. M. M. Ortega, The Burr XII system of densities: properties, regression model and applications, J. Stat. Comput. Simul., 88 (2018), 432-456. 1

[10] V. Couallier, L. Gerville-Réache, C. Huber-Carol, N. Limnios, M. Mesbah, Statistical Models and Methods for Reliability and Survival Analysis, In: Chi-Squared Goodness-of-Fit Test for Normality, 2013 (2013), 213-227. 6

[11] R. V. da Silva, T. A. de Andrade, D. Maciel, R. P. Campos, G. M. Cordeiro, A new lifetime model: The gamma extended Fréchet distribution, J. Stat. Theory Appl., 12 (2013), 39-54. 3.2

[12] B. Efron, Logistic regression, survival analysis, and the Kaplan-Meier curve, J. Amer. Statist. Assoc., 83 (1988), $414-425$.

[13] I. Elbatal, G. Asha, A. V. Raja, Transmuted exponentiated Fréchet distribution: properties and applications, J. Stat. Appl. Prob., 3 (2014), 379-394.

[14] E. J. Freireich, E. Gehan, E. Frei, L. R. Schroeder, I. J. Wolman, R. Anbari, E. O. Burgert, S. D. Mills, D. Pinkel, O.S. Selawry, The effect of 6-mercaptopurine on the duration of steroid-induced remissions in acute leukemia: A model for evaluation of other potentially useful therapy, Blood, 21 (1963), 699-716. 6.3.2

[15] H. Goual, N. Seddik-Ameur, Chi-squared type test for the AFT-generalized inverse Weibull distribution, Comm. Statist. Theory Methods, 43 (2014), 2605-2617. 6

[16] H. Goual, N. Seddik-Ameur, A modified Chi-squared goodness-of-fit test for the kumaraswamy generalized inverse Weibull distribution and its applications, J. Stat. Adv. Theory Appl., 6 (2016), 275-305. 6

[17] P. E. Greenwood, M. S. Nikulin, A guide to chi-squared testing, John Wiley \& Sons, New York, (1996). 6

[18] M. G. Habib, D. R. Thomas, Chi-Square Goodness-if-Fit Tests for Randomly Censored Data, Ann. Statist., 14 (1986), 759-765. 6

[19] D. G. Harlow, Applications of the Fréchet distribution function, Int. J. Mat. Product Tech., 17 (2002), 482-495.

[20] S. Kotz, S. Nadarajah, Extreme Value Distributions: Theory and Applications, Imperial College Press, London, (2000).

[21] E. Krishna, K. K. Jose, T. Alice, M. M. Ristic, The Marshall-Olkin Fréchet Distribution, Comm. Statist. Theory Methods, 42 (2013), 4091-4107. 3.2

[22] M. R. Mahmoud, R. M. Mandouh, On the Transmuted Fréchet Distribution, J. Appl. Sci. Res., 9 (2013), $5553-5561$. 3.2 
[23] M. E. A. Mead, A note on Kumaraswamy-Fréchet Distribution, Aust. J. Basic and Appl. Sci., 8 (2014), 294-300. 3.2

[24] M. Mubarak, Parameter estimation based on the Fréchet Progressive Type II censored data with binomial removals, J. Qual. Statist. Reliab., 2012 (2012), 5 pages.

[25] S. Nadarajah, A. K. Gupta, The Beta Fréchet Distribution, Far East J. Theor. Stat., 14 (2004), 15-24. 3.2

[26] S. Nadarajah, S. Kotz, The exponentiated Fréchet distribution, Interstat Electron. J., 2003 (2003), 1-7. 3.2

[27] M. D. Nichols, W. J. Padgett, A bootstrap control chart for Weibull percentiles, Quality Reliab. Eng. Int., 22 (2006), 141-151. 3.2

[28] M. S. Nikulin, Chi-squared test for continuous distributions with shift and scal parameters, Theory Probab. Appl., 18 (1973), 559-568. 5.1

[29] M. Nikulin, F. Haghighi, A chi-squared test for the generalized power weibull family for the head-and-neck cancer censored data, J. Math. Sci., 133 (2006), 1333-1341.

[30] K. C. Rao, D. S. Robson, A Chi-square statistic for goodness-of-fit tests within the exponential family, Comm. Statist., 3 (1974), 1139-1153. 5.1

[31] R. L. Smith, J. C. Naylor, A comparison of maximum likelihood and Bayesian estimators for the three-parameter Weibull distribution, J. Roy. Statist. Soc. Ser. C, 36 (1987), 358-369. 3.2

[32] R. Varadhan, P. Gilbert, BB: An R package for solving a large system of nonlinear equations and for optimizing a highdimensional nonlinear objective function, J. Statist. Software, 32 (2009), 1-26. 3.3, 5.5.1 